\title{
Functional Analysis of the Transmembrane and Cytoplasmic Domains of Pcdh15a in Zebrafish Hair Cells
}

\author{
Reo Maeda, ${ }^{*}$ Itallia V. Pacentine,* Timothy Erickson, and Teresa Nicolson \\ Oregon Hearing Research Center and the Vollum Institute, Oregon Health and Science University, Portland, Oregon 97239
}

Protocadherin 15 (PCDH15) is required for mechanotransduction in sensory hair cells as a component of the tip link. Isoforms of PCDH15 differ in their cytoplasmic domains (CD1, CD2, and CD3), but share the extracellular and transmembrane (TMD) domains, as well as an intracellular domain known as the common region (CR). In heterologous expression systems, both the TMD and CR of PCDH15 have been shown to interact with members of the mechanotransduction complex. The in vivo significance of these protein-protein interaction domains of PCDH15 in hair cells has not been determined. Here, we examined the localization and function of the two isoforms of zebrafish Pcdh15a (CD1 and CD3) in pcdh15a-null mutants by assessing Pcdh15a transgene-mediated rescue of auditory/vestibular behavior and hair cell morphology and activity. We found that either isoform alone was able to rescue the Pcdh15a-null phenotype and that the CD1- or CD3-specific regions were dispensable for hair bundle integrity and labeling of hair cells with FM4-64, which was used as a proxy for mechanotransduction. When either the CR or TMD domain was deleted, the mutated proteins localized to the stereocilial tips, but were unable to rescue FM4-64 labeling. Disrupting both domains led to a complete failure of Pcdh15a to localize to the hair bundle. Our findings demonstrate that the TMD and cytoplasmic CR domains are required for the in vivo function of Pcdh15a in zebrafish hair cells.

Key words: hair cells; mechanotransduction; PCDH15; tip links; zebrafish

Significance Statement

Tip links transmit force to mechanotransduction channels at the tip of hair bundles in sensory hair cells. One component of tip links is Protocadherin 15 (PCDH15). Here, we demonstrate that, when transgenically expressed, either zebrafish Pcdh15acytodomain 1 (CD1) or Pcdh15a-CD3 can rescue the phenotype of a $p c d h 15 a$-null mutant. Even when lacking the specific regions for $\mathrm{CD} 1$ or $\mathrm{CD} 3$, truncated Pcdh15a that contains the so-called common region (CR) at the cytoplasmic/membrane interface still has the ability to rescue similar to full-length Pcdh15a. In contrast, Pcdh15a lacking the entire cytoplasmic domain is not functional. These results demonstrate that the CR plays a key role in the mechanotransduction complex in hair cells.

\section{Introduction}

Hair cells of the inner ear are specialized for perception of sound, gravity, and head movements in all vertebrates. The hair bundle, which is the mechanically sensitive organelle of a hair cell, is a cluster of actin-filled stereocilia located at the apical surface. Me-

\footnotetext{
Received July 12, 2016; revised Feb. 7, 2017; accepted Feb. 10, 2017

Author contributions: R.M., I.V.P., T.E., and T.N. designed research; R.M., I.V.P., and T.E. performed research; R.M. contributed unpublished reagents/analytic tools; R.M., I.V.P., and T.E. analyzed data; R.M., I.V.P., T.E., and T.N. wrote the paper.

This work was supported by the National Institutes of Health (Grants DC013572 and DC013503), the National Science Foundation (Grant AVPRS0014 to I.V.P.), the Uehara Memorial Foundation, the Naito Foundation, and the Japan Society for the Promotion of Science (JSPS) Postdoctoral Fellowships for Research Abroad (to R.M.).

The authors declare no competing financial interests.

${ }^{*}$ R.M. and I.V.P. contributed equally to this work.

Correspondence should be addressed to Teresa Nicolson, Ph.D., Oregon Hearing Research Center and the Vollum Institute, Oregon Health and Science University, 3181 SW Sam Jackson Park Road L474, Portland, 0R 97239. E-mail: nicolson@ohsu.edu.

DOI:10.1523/JNEUROSCI.2216-16.2017

Copyright $\odot 2017$ the authors $\quad 0270-6474 / 17 / 373231-15 \$ 15.00 / 0$
}

chanical stimuli such as sound pressure and head movement are converted into electrical signals by deflections of the hair bundles along the excitatory axis in a process is called mechanotransduction (Hudspeth, 1989). Extracellular protein filaments known as tip links connect the tips of shorter stereocilia to neighboring taller stereocilia. The tip links are required for mechanotransduction and are thought to transmit physical force to the mechanotransduction channels located at the stereocilia tips (Assad et al., 1991). Although composition may differ during development and regeneration (Ahmed et al., 2006; Indzhykulian et al., 2013), tip links are composed of the heterophilic interaction between cadherin 23 (CDH23) homodimers at the upper end and protocadherin 15 (PCDH15) homodimers at the lower end (Kazmierczak et al., 2007). Because PCDH15 forms the lower portion of the tip link where the mechanotransduction channels are located (Beurg et al., 2009), it is well positioned to interact with other components of the mechanotransduction complex. Indeed, the transmembrane and cytoplasmic domains of PCDH15 have been 
shown to interact with transmembrane channel-like (TMC) proteins (Maeda et al., 2014), Lipoma HMGIC fusion partner-like 5 (LHFPL5) (Xiong et al., 2012), and, in the case of PCDH15-CD2, with transmembrane inner ear (TMIE) (Zhao et al., 2014). Although these interactions are of particular interest when considering how the tip link is tethered to other components of the mechanotransduction complex, it is not clear what domains of PCDH15 are essential for mechanotransduction.

Mammalian hair cells express three isoforms of PCDH15 arising from differential splicing that differ only in the C-terminal cytoplasmic domains (CD1, CD2, and CD3) and share the remaining structures, including the extracellular cadherin (EC) repeats, the transmembrane domain (TMD), and a short stretch of amino acids adjacent to the TMD helix called the "common region" (CR) (Ahmed et al., 2006). PCDH15 isoforms are thought to function redundantly in immature hair cells because isoformspecific knock-out mice lacking PCDH15-CD1, PCDH15-CD2, or PCDH15-CD3 form normal tip links (Webb et al., 2011). However, mice lacking $P C D H 15-C D 2$ are profoundly deaf, whereas hearing is normal in mice lacking $P C D H 15-C D 1$ or $P C D H 15-C D 3$. The hearing loss in mice lacking PCDH15-CD2 was attributed to the loss of kinociliary links in immature cochlear hair cells, causing abnormally polarized hair bundles (Webb et al., 2011). A more recent report showed that the CD2 isoform is required for tip links in mature cochlear hair cells by using specific conditional knock-out mice that lack CD2 after normal hair bundle development. In these mice, the loss of CD2 from mature cells caused profound deafness (Pepermans et al., 2014). Both reports focused on the function of PCDH15 isoforms in cochlear hair cells because vestibular function was unaffected when the isoforms were knocked out individually. Whether the individual isoforms can rescue vestibular function and how the various intracellular regions of PCDH15 contribute to hair bundle integrity and mechanotransduction has not been explored.

In zebrafish, there are two paralogs of $p c d h 15$ : $p c d h 15 a$ is expressed in hair cells and is required for mechanotransduction, whereas $p c d h 15 b$ is expressed in the eye (Seiler et al., 2005). Recently, we reported that there are only two isoforms of Pcdh15a in zebrafish, Pcdh15a-CD1 and Pcdh15a-CD3. The Pcdh15a-CD2 isoform is not detectable at either the transcript level nor within the genomic region of pcdh15a (Seiler et al., 2005; Maeda et al., 2014). As in mammals, the zebrafish CD1 and CD3 isoforms share the extracellular cadherin repeats, the transmembrane domain, and the intracellular common region. To test the function of the zebrafish Pcdh15a isoforms in hair cells, we quantified the ability of wild-type and mutated versions of $p c d h 15 a$ transgenes to rescue defects in behavior, hair bundle morphology, and activity in pcdh15a-null mutants.

\section{Materials and Methods}

Zebrafish husbandry. Zebrafish (Danio rerio) were maintained at $28^{\circ} \mathrm{C}$ and bred using standard conditions. Animal research complied with guidelines stipulated by the Institutional Animal Care and Use Committee at Oregon Health and Science University. In this study, the following zebrafish mutant alleles were used: $p c d h 15 a^{\text {th263b }}$ (Nicolson et al., 1998; Seiler et al., 2005), $\operatorname{lhfp} 15 a^{\operatorname{tm} 290 \mathrm{~d}}$ (Obholzer et al., 2008), myo6b $b^{\operatorname{tn} 3137}$ (Seiler et al., 2004), $c d h 23^{\text {n19 }}$ (Obholzer et al., 2008), and myo7aa ${ }^{\text {ty220d }}$ (Ernest et al., 2000). All lines in this study were maintained in a Tübingen or Top long fin wild-type background. For experiments, we used larvae at 4-6 d postfertilization ( $\mathrm{dpf}$ ), which are of indeterminate sex at this stage.

Plasmid construction and transgenic lines. The Tol2/Gateway system was used to make expression vectors (Kwan et al., 2007). pcdh15a-CD1 (GenBank accession number AY772390), pcdh15a-CD3 (KY432405), and engineered variants were subcloned using restriction enzymes and ligation or the in-fusion method into the middle entry vector, with the monomeric EGFP gene fused to the $\mathrm{C}$ terminus. In these constructs, the flexible linker "(GGGGS)" was introduced between the $p c d h 15 a$ variants and EGFP (Chen et al., 2013). Standard Gateway LR reactions were performed to generate the following constructs: pDest(-6myo6b:pcdh15a-CD1-EGFP-pA), pDest(-6myo6b-pcdh15a-CD3-EGFP-pA), pDest(-6myo6b-pcdh15a-CREGFP-pA), pDest(-6myo6b-pcdh15a( $\Delta c y t o)-E G F P-p A)$, pDest(-6myo6b$p c d h 15 a(C D 8 T M D)-C R-E G F P-p A)$, and pDest(-6myo6b-pcdh15a(CD8 $T M D)(\Delta c y t o)-E G F P-p A)$. Each construct contained either a GFP or Redstar heart marker for transgenesis. To generate transgenic fish, plasmid DNA and tol2 transposase mRNA were coinjected into eggs collected from th263b heterozygous fish; injections were done at the one-cell stage as described previously (Kwan et al., 2007). More than 500 eggs were injected for each construct and five or more founders were analyzed. The founders with the brightest signal in the hair bundles were selected for breeding. Founders were bred with th $263 b$ heterozygous or wild-type homozygous fish. To obtain th $263 b$ heterozygous fish carrying an insertion, heterozygous founders were crossed to nontransgenic heterozygous fish and expression of the GFP fusion protein was ascertained by confocal microscopy; the genotype of the adult fish was confirmed by performing PCR with DNA isolated from fin clips. To generate th $263 b$ homozygous adults with inserts, transgenic heterozygous fish were crossed to nontransgenic heterozygous fish; the resulting progeny were raised to adulthood and genotyped. Either heterozygous or homozygous transgenic fish were crossed to nontransgenic heterozygous fish for the auditory escape behavioral response (AEBR) and FM-labeling experiments.

In situ hybridization and semiquantitative PCR. The CD1- or CD3specific template for in vitro transcription was amplified by PCR using primers containing promoter sequence (T7/T3 promoter). The following pairs of primers were used: T3-CD1 forward, CATTAACCCTCACTAAAGGGAA ATGCTTGGACTACAAAGACG and T7-CD1 reverse, TAATACGACTCACTATAGGGTTATACATCGTTCTTGTTGT for CD1 specific region (1017 bp); T3-CD3 forward, CATTAACCCTCACTAAAGGGAAATCAG GCGGGGCATGGGCAG and T7-CD3 reverse, TAATACGACTCACTATAGGGTCAGAGTTTTGTCATTGGTA for CD3 specific region (807 bp). Digoxigenin (DIG)-labeled sense or antisense RNA probes were synthesized by using DIG labeling mix (Roche) and T7 or T3 RNA polymerase (Promega). In situ hybridization was performed as described previously (Thisse and Thisse, 2008; Erickson et al., 2010). Specimens were mounted on a depression slide in 1.2\% low-melting-point agarose and imaged on a Leica DMLB microscope fitted with a Zeiss AxioCam MRc 5 camera using Zeiss AxioVision acquisition software (version 4.5).

For semiquantitative PCR, cDNA was prepared from RNA isolated from the maculae of adult inner ears. The following primers were used: pcdh15aCD1 forward, ATCCAGATGGCACTTCCTGC; pcdh15a-CD1 reverse, CCGCCTCCTCGATTACAGAC, predicted product at $163 \mathrm{bp}$; pcdh15aCD3 forward, GGAGGCTGATCACTCCGATG; pcdh15a-CD3, reverse, GTGACTGCTGACGTTGGGTA, predicted product at $197 \mathrm{bp}$. Lower and higher weight amplicons represent different splice variants (Maeda et al., 2014). The PCRs were run through 25, 30, and 35 cycles. The resulting PCR products from the 35 cycle reaction (all three bands for CD1 and two bands for CD3) were quantified using ImageJ software.

Immunofluorescent staining and microscopy. To immunolabel hair bundles of the inner ear with anti-Pcdh15a monoclonal antibody directed against an N-terminal fragment of Pcdh15a (1-324 aa; Maeda et al., 2014), larvae (4 or $6 \mathrm{dpf}$ ) were fixed with $4 \%$ formaldehyde in PBST $\left(0.01 \%\right.$ Tween 20 in PBS) overnight at $4^{\circ} \mathrm{C}$ and rinsed with PBST. Larvae were permeabilized in $0.5 \%$ Triton X-100 in PBS for $1 \mathrm{~h}$ with $50 \mathrm{rpm}$ shaking at room temperature (RT), then at $4^{\circ} \mathrm{C}$ overnight without shaking. They were then incubated in blocking solution (PBS/1\% BSA $/ 5 \%$ goat serum) for $2 \mathrm{~h}$, followed by overnight incubation with primary antibody at $4^{\circ} \mathrm{C}$. The Pcdh15a antibody was used at 1:200 in blocking solution. Alexa Fluor 546-conjugated goat anti-mouse IgG (Life Technologies) was used at 1:500 for $4-5 \mathrm{~h}$ at RT. Rhodamine-phalloidin (Life Technologies) was used at 1:50 with the secondary antibody in order to visualize actin filaments in hair bundles.

For imaging of live and fixed samples with confocal microscopy, an upright Zeiss LSM700 laser-scanning confocal microscope with a Plan Apochromat $40 \times / 1.0$ differential interference contrast (DIC) or Acroplan $63 \times / 0.95 \mathrm{~W}$ lens was used. Live larvae were anesthetized with E3 
plus 0.03\% 3-amino benzoic acid ethylester (MESAB; Western Chemical) and live or fixed larvae were mounted in low-melting-point agarose (Sigma-Aldrich).

FM4-64 labeling of neuromast hair cells. To investigate the basal activity of neuromast hair cells, zebrafish larvae ( 4 or $6 \mathrm{dpf}$ ) were incubated for $30 \mathrm{~s}$ in E3 medium containing $3 \mathrm{~mm} \mathrm{~N}$ [SCAP]-(3-triethylammoniumpropyl)-4-(6(4-(diethylamino)phenyl)hexatrienyl)pyridinium dibromide (FM4-64; Life Technologies). After treatment, larvae were washed with E3 3 times and then anesthetized with E3 plus 0.03\% MESAB. For each experiment, microscope parameters were adjusted to avoid saturation of the pixels. The levels of FM4-64 labeling were quantified using ImageJ software. First, maximum projections of the neuromasts were made using seven optical sections beginning at the cuticular plate and moving down so as to include the cell bodies and exclude the bundles. The mean pixel value of each maximum projection was then calculated in the channel with an emission peak at $640 \mathrm{~nm}$. The number of hair cells was determined by counting the somas within the hair cell layer in the DIC images. The mean pixel value was divided by the number of cells, yielding a mean pixel value/cell for each neuromast. The mean value/ cell of each wild-type dataset was set to 1.0; each mutant value/cell was then normalized to the corresponding sibling wild-type mean.

Behavioral assays. Quantification of vestibular-induced eye movements was performed in larvae using a method described previously (Mo et al., 2010). In brief, zebrafish larvae at $5 \mathrm{dpf}$ were mounted in $2 \%$ low-melting-point agarose in E3 medium on a coverslip and agarose around the head was removed to free the eyes. This space was filled with E3 medium. Larvae were mounted on the specimen platform of the device in a head-down position perpendicular to the platform. During the rotation of the platform at $0.25 \mathrm{~Hz}$, eye movements were recorded with ScopePhoto and the recorded data were processed in MATLAB (The MathWorks).

Monitoring of the AEBR was quantified by using a method similar to one described previously (Einhorn et al., 2012). Larvae were presorted based on EGFP fluorescence or genotyped afterward for the presence of the fusion protein using primers directed against EGFP (forward: ACG TAAACGGCCACAAGTTC and reverse: AAGTCGTGCTGCTTCATG TG). Briefly, larvae at $5 \mathrm{dpf}$ were placed in the central 6 wells of a 96-well microplate, in the dark inside a Zebrabox monitoring system (ViewPoint Life Sciences) and stimulated with twelve $100 \mathrm{~ms}$ pure-tone stimuli (1 $\mathrm{kHz}$ at a sound pressure level of $157 \mathrm{~dB}$ to elicit a $>50 \%$ response in control larvae) spaced at $15 \mathrm{~s}$ intervals for $3 \mathrm{~min}$. If spontaneous movements occurred within $1 \mathrm{~s}$ before the stimulus, then that interval was omitted from analysis (Fig. $4 A f$, blue asterisks). If spontaneous movements occurred during more than six stimuli, then those larvae were omitted from subsequent analysis. Each larva was subjected to one trial; $13.3 \%(16 / 120)$ of the wild-type larvae were not responsive, resulting in a non-Gaussian distribution of the data. Larvae were genotyped afterward for the $t h 263 b$ mutation (forward: GGCACACCTTCTACGTACCC and reverse: ACGCTCAAATAACGGTGAGC primers).

\section{Results \\ Both isoforms of Pcdh15a (CD1 and CD3) localize at the tips of hair bundles}

Previously, we reported that zebrafish $p c d h 15 a$ is expressed in hair cells of the inner ear and lateral line organ, and in regions of the larval brain, using an antisense RNA probe that recognized both isoforms without differentiating between the two (Seiler et al., 2005). To determine the spatial and temporal expression of each isoform individually, we used probes against the CD1- or CD3-specific coding regions (Fig. $1 A$ ). At $4 \mathrm{dpf}$, both splice variants are expressed in the hair cells of the lateral line organ and inner ear (Fig. $1 B-G$ ), as well as in the brain (Fig. $1 B, E$ ). Compared with $p c d h 15 a-C D 1$ expression, $p c d h 15 a-C D 3$ appears to be expressed at higher levels in hair cells (Fig. $1 C, D, F, G$ ). To assess whether the CD3 isoform is expressed at higher levels in zebrafish hair cells, we performed semiquantitative PCR using cDNA generated from adult inner ears. Consistent with the in situ data and previous reports on larval $p c d h 15 a$ transcripts (Sheets et al., 2011;
Maeda et al., 2014), we observed that the level of pcdh15a-CD3 mRNA in adult ears was $>2$-fold higher than $p c d h 15 a-C D 1$ transcripts (Fig. 1H; normalized to the gapdh control, the relative densities are 0.7 for $C D 3$ and 0.3 for CD1 PCR products; multiple bands representing the different splice variants were included; Maeda et al., 2014).

Our previous study showed that endogenous Pcdh15a is localized at the tips of zebrafish hair bundles (Maeda et al., 2014). However, immunolabeling of individual isoforms was not possible because our monoclonal antibody recognizes an $\mathrm{N}$-terminal antigen in Pcdh15a (Fig. $2 A$, black bar, $B-B^{\prime \prime \prime}$ ). We therefore determined the localization of Pcdh15a-CD1 and Pcdh15a-CD3 in zebrafish hair cells by generating transgenic zebrafish expressing either Pcdh15aCD1 or Pcdh15a-CD3 tagged with monomeric EGFP and driven by the hair-cell-specific myosin $6 b$ (myo6b) promoter (Fig. $2 A)$. We imaged inner ear hair cells within lateral cristae at $6 \mathrm{dpf}$. At this stage, the epithelium includes mainly mature cells bordered by a few immature hair cells. Hair cells were identified as immature based on two criteria: (1) their peripheral position within the epithelium and (2) the shorter height of their hair bundles (Kindt et al., 2012). In more mature hair cells, subcellular localization of GFP-tagged Pcdh15a-CD1 and Pcdh15a-CD3 was detected in a punctate pattern at the tips of hair bundles (Fig. $\left.2 C-D^{\prime \prime \prime}\right)$. Importantly, this organized pattern of exogenously expressed Pcdh15a is nearly identical to that seen with immunolabeling for the endogenous Pcdh15a (Fig. $2 B$ $\left.B^{\prime \prime \prime}\right)$. A less punctate pattern was observed in immature hair cells at the periphery of the neuroepithelium (Fig. 2D, yellow arrowheads), although the signal appeared to be concentrated near the tip of these shorter hair bundles. The increase in density and broader distribution of PCDH15 in immature hair cells [embryonic day 9 (E9)-E12] was also reported in the chick basilar papilla (Goodyear and Richardson, 2003). Together, these results suggest that GFP-tagged versions of both Pcdh15a isoforms localize in a similar fashion to endogenous Pcdh15a and are components of tip links in zebrafish hair cells.

Pcdh15a-EGFP localization in mechanotransduction mutants Studies in fish and mice have identified several genes implicated in mechanotransduction and the integrity of hair bundles. Some of these genes, such as Cdh23, Lhfpl5, and Myo7a, have been shown to affect the localization of PCDH15 in stereocilia (Senften et al., 2006; Xiong et al., 2012). To determine whether EGFPtagged Pcdh15a protein also exhibits the same requirements for localization, we imaged Pcdh15a-CD3-EGFP in $\operatorname{lhfpl} \mathrm{a}^{\mathrm{tm} 290 \mathrm{~d}}$, $c d h 23^{\text {nl9 }}$, and myo7aa ${ }^{\text {ty220d }}$ mutant hair cells. As a control, we also examined a fourth mechanotransduction mutant that is not known to have a direct effect on the transduction complex, but rather affects bundle integrity, the myosin $6 b\left(\right.$ myo6 $\left.^{\operatorname{tn} 3137}\right)$ mutant.

LHFPL5 (previously known as TMHS) is a component of the mechanotransduction complex in mouse cochlear hair cells (Xiong et al., 2012). Mutations in LHFPL5 cause nonsyndromic deafness in humans and auditory/vestibular defects in mice and fish (Longo-Guess et al., 2005; Shabbir et al., 2006). Previous data showed that LHFPL5 is required for localization of PCDH15 to hair bundles and vice versa (Xiong et al., 2012). To first compare whether changes in localization of the tagged version of Pcdh15a were similar to perturbations in endogenous Pcdh15a, we examined immunolabeling of Pcdh15a in lhfpl5a $a^{\text {tm290d }}$ mutants. As reported in mouse Lhfpl5 ${ }^{-1-}$ cochlear hair cells, we observed that immunolabeling of Pcdh15a in hair bundles was greatly reduced in zebrafish $l h f p l 5 a^{\mathrm{tm} 290 \mathrm{~d}}$ mutants (Fig. $3 B, B^{\prime}$ ) compared to wild-type siblings (Fig. 
A

\section{Pcdh15aCD1}

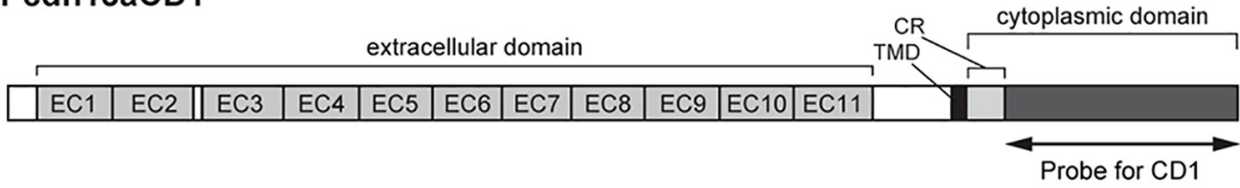

\section{Pcdh15aCD3}
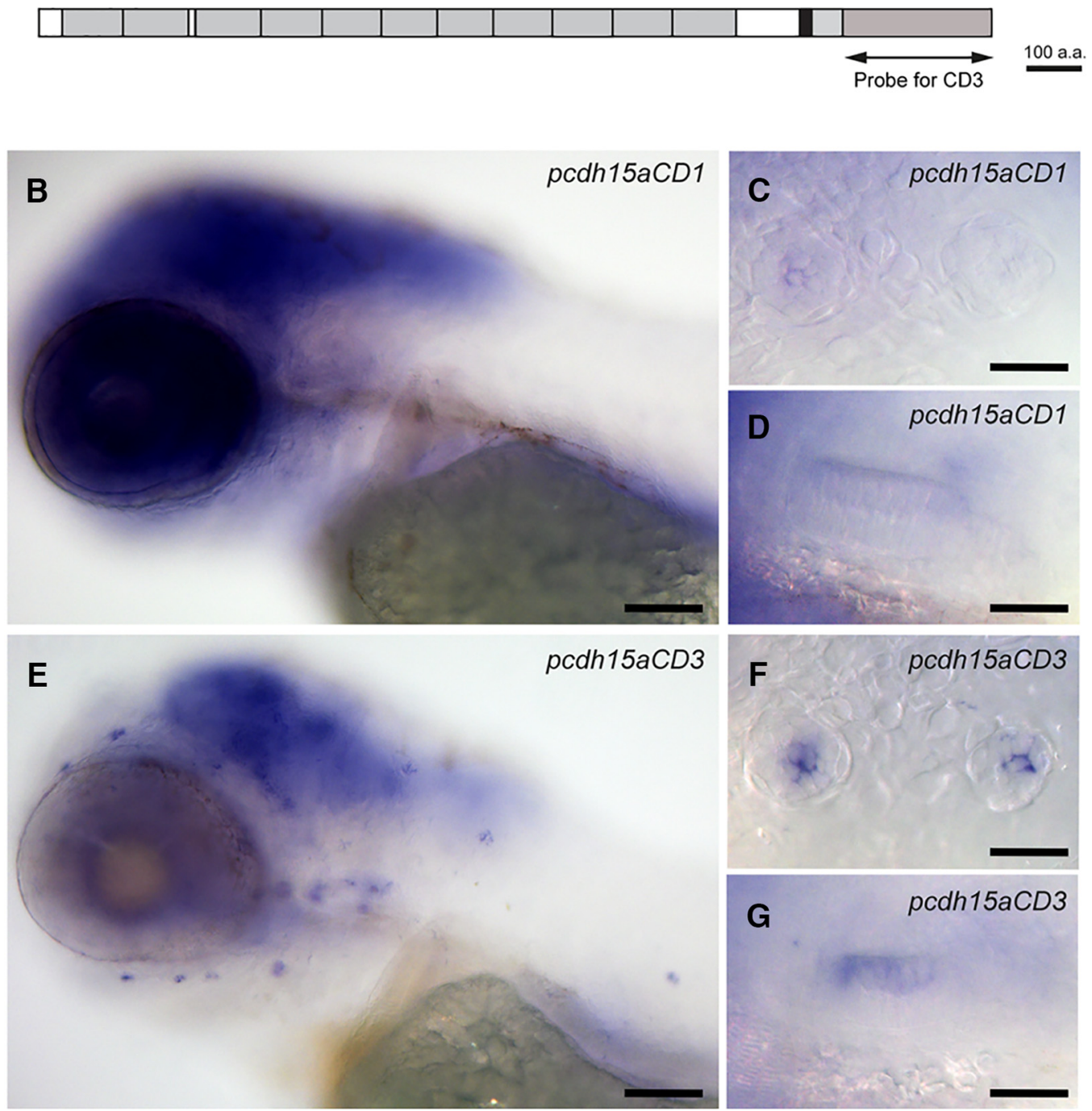

H

adult inner ear

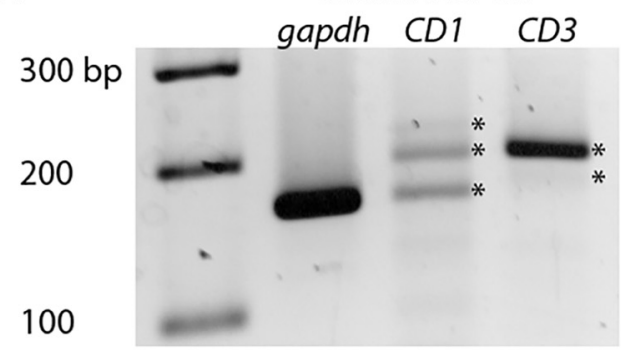

Figure 1. Expression pattern of the two splice variants $p c d h 15 a-C D 1$ and $p c d h 15 a-C D 3$ using in situ hybridization with specific probes for each transcript. $A$, Schematic drawing of $P c d h 15$ a-CD1 and $P$ cdh 15a-CD3 proteins. The positions of the probes used for in situ hybridization experiments are indicated. $\boldsymbol{B}-\boldsymbol{D}, p c d h 15 a-C D 1$ expression at 4 dpf. $\boldsymbol{E}-\boldsymbol{G}, p c d h 15 a-C D 3$ expression at 4 dpf. $\boldsymbol{C}, \boldsymbol{F}$, Higher-magnification views of two trunk neuromasts. $\boldsymbol{D}, \mathbf{G}$, Higher-magnification view of the anterior macula. Scale bars: $\boldsymbol{B}, \boldsymbol{E}, 100 \mu \mathrm{m} ; \boldsymbol{C}, \boldsymbol{D}, \boldsymbol{F}, \mathbf{G}, 25 \mu \mathrm{m}$. $\boldsymbol{H}$, Semiquantitative PCR of $p c d h 15$ a cDNA from adult inner ear tissue using isoform-specific primers (products from 35 cycles are shown). The relative density of the PCR products for CD3 splice variants ( 2 bands) is 2.3 -fold higher than CD1 splice variants (three bands). Bands are indicated by asterisks. 
A

\begin{tabular}{|c|c|c|c|c|c|c|c|c|c|c|}
\hline Pcdh1 & $\mathrm{aCD}$ & -EGF & & extrac & ellular & domai & & & cytoplasmic domain & \\
\hline EC1 & EC2 & EC3 & EC4 & EC5 & EC6 & EC7 & EC8 & \begin{tabular}{|l|l|l|} 
EC9 & EC10 & EC11 \\
\end{tabular} & & EGFP \\
\hline
\end{tabular}

\section{Pcdh15aCD3-EGFP}
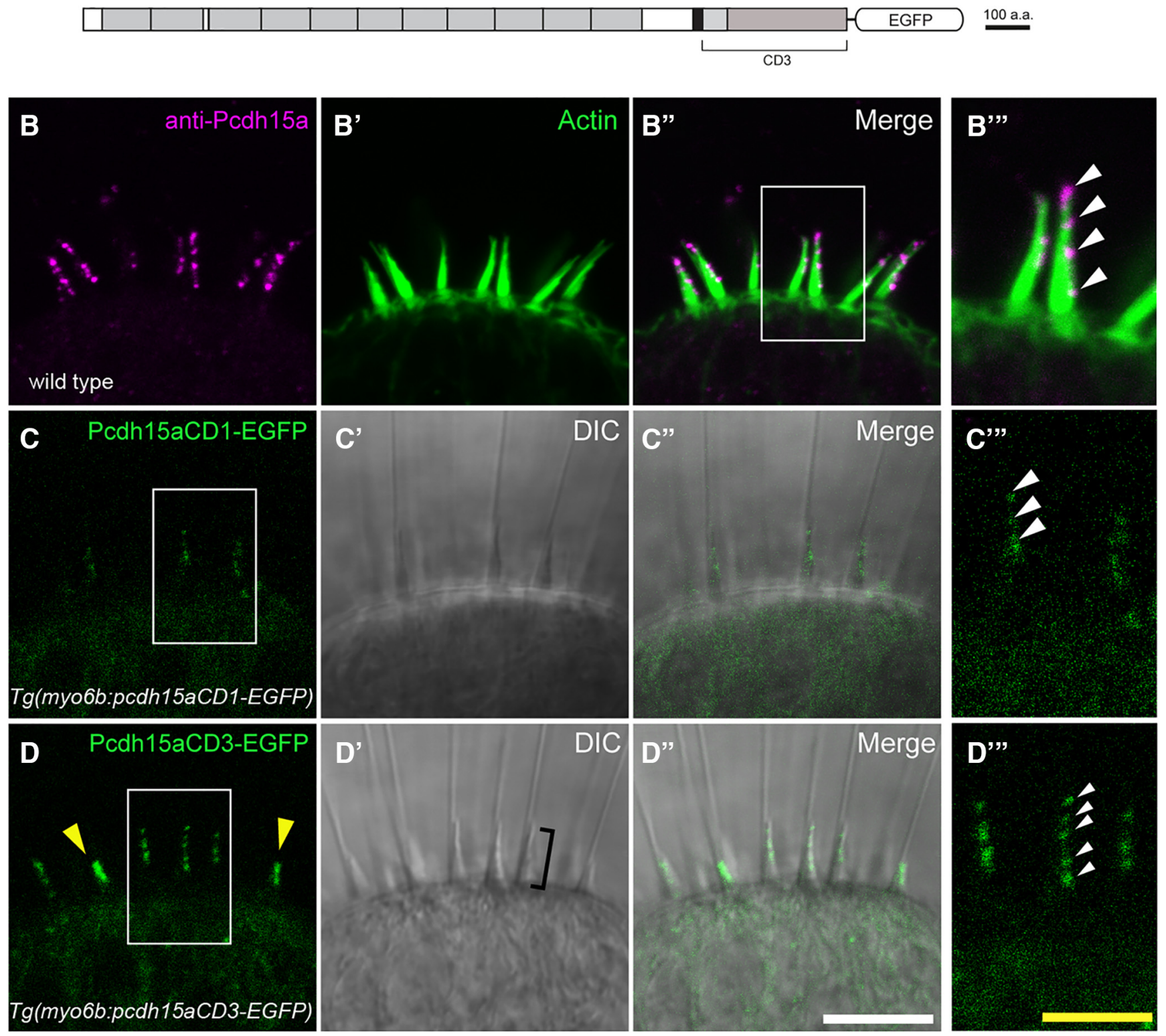

Figure 2. Imaging of EGFP-tagged Pcdh15a isoforms in vestibular hair cells of live fish. $A$, Schematic of zebrafish Pcdh15a-CD1 or Pcdh15a-CD3 fused at its $C$ terminus to EGFP. The antigen used to generate the antibody is indicated by the black bar. $\boldsymbol{B}-\boldsymbol{D}^{\prime \prime \prime}{ }^{\prime}$, Representative confocal images of wild-type hair cells in the lateral cristae of inner ear at $6 \mathrm{dpf} . \boldsymbol{B}-\boldsymbol{B}^{\prime \prime}{ }^{\prime}$, Pcdh15a antibody label (magenta) of the lateral crista at $6 \mathrm{dpf}, z$ projection. To visualize the hair bundles, actin (green) was labeled using phalloidin $\left(\boldsymbol{B}^{\prime}\right)$. $\boldsymbol{C}-\boldsymbol{C}^{\prime \prime}{ }^{\prime}$, Image of the localization pattern of Pcdh15a-CD1-EGFP in a stable transgenic line, $z$ projection. $\boldsymbol{D}-\boldsymbol{D}^{\prime \prime \prime}$ ', Localization pattern of Pcdh15a-CD3-EGFP in a stable transgenic line, single section. Yellow arrowheads indicate two immature hair bundles. Bracket in $\boldsymbol{D}^{\prime}$ indicates hair bundle. $\boldsymbol{B}^{\prime \prime \prime}, \boldsymbol{C}^{\prime \prime \prime}, \boldsymbol{D}^{\prime \prime \prime}$, Higher-magnification image of area from $\boldsymbol{B}^{\prime \prime}, \boldsymbol{C}$, and $\boldsymbol{D}$, respectively (outlined with box). Note the staircase-like localization at the tip of the hair bundles. White scale bars in $\boldsymbol{B}-\boldsymbol{B}^{\prime \prime}, \boldsymbol{C}-\boldsymbol{C}^{\prime \prime}, \boldsymbol{D}-\boldsymbol{D}^{\prime \prime}, 10 \mu \mathrm{m}$. Yellow scale bars in $\boldsymbol{B}^{\prime \prime \prime}, \boldsymbol{C}^{\prime \prime \prime}, \boldsymbol{D}^{\prime \prime \prime}, 5 \mu \mathrm{m}$.

$\left.3 A, A^{\prime}\right)$. We next examined the localization pattern of stably expressed Pcdh15a-CD3-GFP in $\operatorname{lhfpl} 5 a^{\mathrm{tm} 290 \mathrm{~d}}$ mutants and found that Pcdh15a-CD3-EGFP was largely absent in both mature and immature hair bundles, but detectable in hair cell somas (Fig. $3 C-C^{\prime \prime \prime}$ ). This pattern is similar to that seen with endogenous label in Figure 3, $B$ and $B^{\prime}$, and to the pattern observed with anti-PCDH15 antibodies in Lhfpl5 ${ }^{-1-}$ mice (Xiong et al., 2012). One difference is that there were higher levels of tagged Pcdh15a in the cell bodies of relatively mature hair cells in $l h f p l 5 a^{\operatorname{tm} 290 \mathrm{~d}}$ mutants. This difference is likely due to exogenous expression of Pcdh15a. Nonetheless, the strong reduction of immunolabeling and Pcdh15a-CD3-EGFP in 

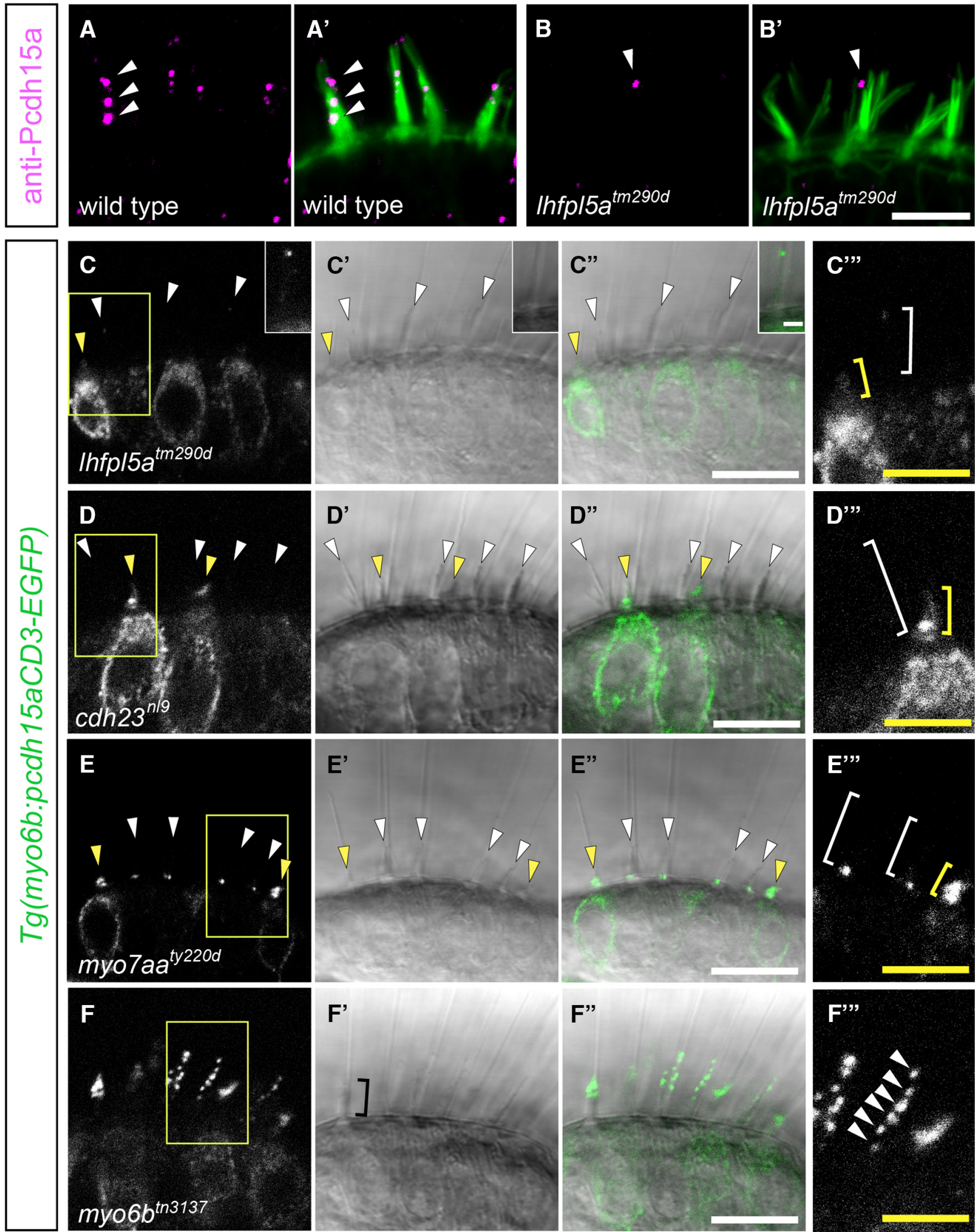

Fry
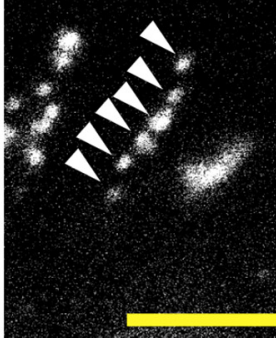

Figure 3. Localization of Pcdh15a in mechanotransduction mutants. Immunolabel of Pcdh15a (magenta) and phalloidin-labeled actin (green) in a wild-type sibling (A, $\left.\boldsymbol{A}^{\prime}\right)$ and $/ h f p / 5 a^{\text {tm290d }}$ mutant $\left(\boldsymbol{B}, \boldsymbol{B}^{\prime}\right)$. An example of remaining signal in a mutant hair bundle is shown (arrowhead). $\boldsymbol{C}-\boldsymbol{F}^{\prime \prime}{ }^{\prime}$, images of stably expressed EGFP-tagged Pcdh15a-CD 3 in mechanotransduction mutants at $6 \mathrm{dpf}$. Representative confocal images are shown. The white arrowheads and brackets indicate mature hair bundles, whereas the yellow arrowheads and brackets indicate immature hair bundles at the periphery of the cristae. $C_{-} C^{\prime \prime}{ }^{\prime \prime}, I h f p l 5 a^{\text {tm290d }}$ mutant; Pcdh15a-CD3-EGFP localized predominantly to the soma, but faint signal was occasionally detectable at the tips of the longest stereocilia

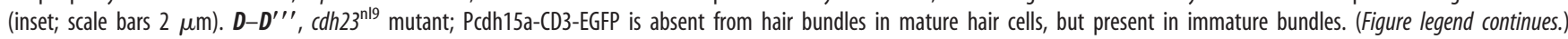


mutant hair bundles suggests that the Pcdh15a-CD3-EGFP protein behaves similarly to endogenous Pcdh 15 a. Having confirmed that our transgene expression closely matches that of endogenous protein, we performed the rest of our experiments using Pcdh15a-CD3-EGFP.

$\mathrm{CDH} 23$ has been shown to be a component of tip links in mice and fish (Söllner et al., 2004; Siemens et al., 2004); CDH23 homodimers interact in trans with PCDH15 homodimers to form tip links (Kazmierczak et al., 2007). Moreover, disrupting tip links with $\mathrm{Ca}^{2+}$ chelators results in the absence or redistribution of PCDH15 in chick and mouse hair cells (Goodyear and Richardson, 2003; Indzhykulian et al., 2013). In zebrafish $c d h 23^{\text {n19 }}$ mutants, Pcdh15a-CD3-EGFP was present in the cell bodies of inner ear hair cells, whereas accumulation of Pcdh15a-CD3EGFP in hair bundles was only observed in immature hair cells (Fig. $3 D-D^{\prime \prime \prime}$, yellow arrows). This result indicates that Cdh23 is required for the stable localization of Pcdh15a-CD3-EGFP at the tips of hair bundles.

In addition to LHFPL5 and CDH23, MYO7A has also been shown to interact with PCDH15 (Senften et al., 2006). Like Myo7a mutant mice, we observed that Pcdh15a-CD3-EGFP was absent from the tips of mature hair bundles in the orthologous zebrafish myo7aa ${ }^{\text {ty220d }}$ mutant (Fig. $3 E-E^{\prime \prime \prime}$, white arrowheads and brackets); however, GFP signal was present near the base of the mature hair bundles. As in $c$ dh 23 mutants, we detected GFP signal in immature bundles (Fig. $3 E-E^{\prime \prime \prime}$, yellow arrowheads and bracket), indicating that Myo7aa is not required for trafficking of Pcdh15a into hair bundles, but rather is necessary for stable localization of Pcdh15a at the tips of stereocilia. Collectively, the results in the $c d h 23, \operatorname{lhfpl5a}$, and myo7aa mutant backgrounds suggest that GFP-tagged Pcdh15a is behaving as predicted by previous studies and that the interactions among these proteins are conserved in zebrafish.

Zebrafish Myo6b has been shown to be required for maintaining the structural integrity of the apical surface of zebrafish hair cells, presumably by regulating actin-based interactions with the plasma membrane (Seiler et al., 2004). In myo6b mutants, hair bundles show multiple phenotypes: they can be splayed, misshapen, or exhibit fusion of the stereocilia, similar to the phenotype seen in Snell's waltzer mice (Seiler et al., 2004). We found that in $m y o 6 b^{\text {tn3137 }}$ mutants, Pcdh15a-CD3-EGFP was still distributed to the tips of stereocilia in a punctate manner, even in splayed bundles (Fig. $3 F-F^{\prime \prime \prime}$, arrowheads). In fused bundles (Fig. $3 F^{\prime}$, black bracket), the localization at stereocilial tips resulted in Pcdh15a-CD3-EGFP puncta that were more intense than in wild-type. This result suggests that the activity of Myo6b and the integrity of the hair bundle are not required for localization of Pcdh15a.

\section{Both isoforms of Pcdh15a rescue hearing and balance deficits in pcdh15a mutants}

Within $4 \mathrm{~d}$ of development, the zebrafish inner ear is functional, allowing larvae to maintain an upright position and exhibit a robust startle reflex to acoustic stimuli. Larvae carrying muta-

\section{$\leftarrow$}

(Figure legend continued.) $\quad \boldsymbol{E}-\boldsymbol{E}^{\prime \prime \prime}{ }^{\prime}$, myo7aa ${ }^{\text {ty220d }}$ mutant; Pcdh15a-CD3-EGFP was absent in hair bundles of mature cells, similar to $c d h 23$ mutants. $\boldsymbol{F}-\boldsymbol{F}^{\prime \prime}{ }^{\prime}$, myo6 $b^{\text {th3137 }}$ mutant; Pcdh15aCD3-EGFP localized at the tips of hair bundles in a staircase pattern (arrowheads in $\boldsymbol{F}^{\prime \prime}{ }^{\prime \prime}$ ). Black bracket in $\boldsymbol{F}^{\prime}$ indicates a fused bundle phenotype. $\boldsymbol{C}^{\prime \prime \prime}, \boldsymbol{D}^{\prime \prime \prime}, \boldsymbol{E}^{\prime \prime}{ }^{\prime}, \boldsymbol{F}^{\prime \prime}{ }^{\prime}$, Higher-magnification image of area from $\boldsymbol{C}-\boldsymbol{F}$, respectively (outlined with yellow box). $n>5$ larvae for each genotype. Scale bars in $\boldsymbol{C}-\boldsymbol{C}^{\prime \prime}, \boldsymbol{D}-\boldsymbol{D}^{\prime \prime}, \boldsymbol{E}-\boldsymbol{E}^{\prime \prime}, \boldsymbol{F}-\boldsymbol{F}^{\prime \prime}, 10 \mu \mathrm{m}$. Scale bars in $\boldsymbol{A}-\boldsymbol{B}^{\prime}, \boldsymbol{C}^{\prime \prime \prime}, \boldsymbol{D}^{\prime \prime \prime}, \boldsymbol{E}^{\prime \prime \prime}$, $F^{\prime \prime \prime}, 5 \mu \mathrm{m}$. tions in $p c d h 15 a$ have pronounced auditory and vestibular defects (Granato et al., 1996; Nicolson et al., 1998). To explore whether the expression of a single isoform is sufficient for restoring hearing and balance, we performed rescue experiments in $p c d h 15 a^{\text {th2 } 63 \mathrm{~b}}$ mutants. The th $263 \mathrm{~b}$ mutation is a null allele that causes a severe truncation of the Pcdh15a protein within the third extracellular cadherin repeat (R360X), thus affecting both CD1 and CD3 isoforms and leading to a complete loss of microphonic potentials and mechanically evoked calcium transients in hair cells (Nicolson et al., 1998; Seiler et al., 2005; Kindt et al., 2012). First, we sought to quantify auditory function by comparing the AEBR in wild-type and $p c d h 15 a^{\text {th263b }}$ mutants. We assessed this behavior by exposing the larvae to pure tone stimuli $(157 \mathrm{~dB}$, $1000 \mathrm{~Hz}$ for $100 \mathrm{~ms}$ ) delivered at regular $15 \mathrm{~s}$ intervals for $3 \mathrm{~min}$ and quantifying the resulting acoustically evoked movements (Fig. 4A; red indicates large movements above the background movements shown in green). As expected, wild-type larvae showed a robust response to acoustic stimuli (Fig. 4Aa,B), whereas $p c d h 15 a^{\text {th263b }}$ mutants had almost no response to the tones (Fig. $4 A b, B$ ). Upon stable transgenic expression of either full-length isoform of Pcdh15a, we observed that startle responses to the tones were restored (Fig. $4 A c, A d, B$ ). In each case, the rescue of hearing deficits was comparable: the startle responses among homozygous mutants expressing the transgenes were not statistically different from the responses of their respective transgenic heterozygous siblings (Fig. $4 B$ ). We also noted that, regardless of genotype, transgenic fish expressing either fulllength isoform often exhibited an increase in spontaneous, nonauditory evoked movements. It is not clear why expression of the transgenes would cause hyperactivity and these fish were excluded from our AEBR analysis (Fig. 4Af). Nevertheless, these results indicate that either isoform is capable of rescuing auditory deficits in pcdh15a-null mutants.

Next, we quantified the rescue of vestibular function by testing vestibular-induced eye movements. These reflexive movements in zebrafish larvae occur in response to rotation of the head and consequent stimulation of the anterior maculae (Mo et al., 2010). Wild-type larvae moved their eyes robustly in response to rotation (Fig. 4C). In contrast, $p c d h 15 a^{\text {th263b }}$ mutants did not have detectable eye movements during rotation (Fig. 4C). Stable expression of a $p c d h 15 a-C D 1$ or $p c d h 15 a-C D 3$ transgene significantly rescued the vestibular deficits in $p c d h 15 a^{\text {th263b }}$ mutants compared with wild-type siblings (Fig. 4C). These behavioral experiments suggest that, when expressed exogenously, either Pcdh $15 \mathrm{a}$ isoform is capable of restoring the activity of the auditory/vestibular organs in a pcdh15a-null mutant.

\section{Both isoforms of Pcdh15a rescue splayed hair bundles in pcdh15a mutants}

Previously, we reported that $p c d h 15 a$ is required for the integrity of hair bundles in the inner ear (Seiler et al., 2005). Similar to live images of inner ear hair cells in $l h f p l 5 a$ and $c d h 23 \mathrm{mu}$ tant larvae (Fig. $3 C^{\prime}, D^{\prime}$ ), hair bundles in $p c d h 15 a^{\text {th } 263 \mathrm{~b}}$ mutant larvae are disorganized and splayed, with individual or clusters of stereocilia separated from the bundle or kinocilia (Fig. $5 B$, arrowheads, Fig. $5 A$, wild-type hair bundles). We investigated whether transgenic expression of $p c d h 15 a-C D 1-E G F P$ or pcdh15a-CD3-EGFP could rescue the morphological defects in $p c d h 15 a^{\text {th263b }}$ mutant larvae. In intact larvae, we observed that both GFP-tagged isoforms were localized to the tips of mutant hair bundles and were able to rescue the abnormal splaying of stereocilia (Fig. 5C,D). 
A

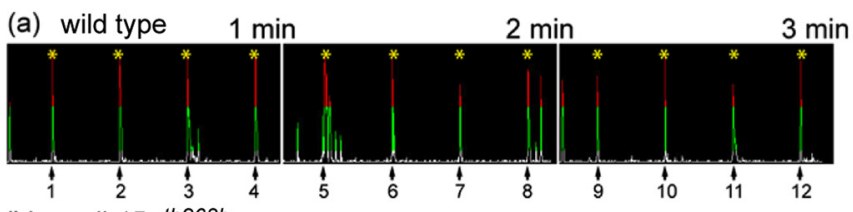

(b) pcdh15ath263b

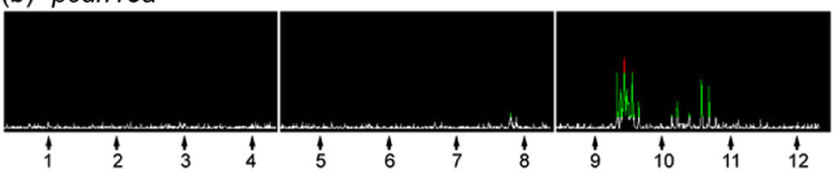

(c) $\operatorname{Tg}$ (myo6b:pcdh15aCD1-EGFP); pcdh15ath263b

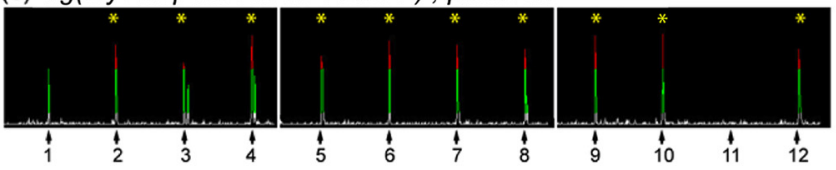

(d) $\operatorname{Tg}($ myo6b:pcdh15aCD3-EGFP) ; pcdh15ath263b

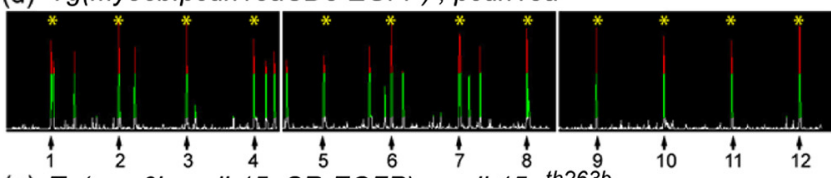

(e) $\operatorname{Tg}($ myo6b:pcdh15aCR-EGFP) ; pcdh15ath263b

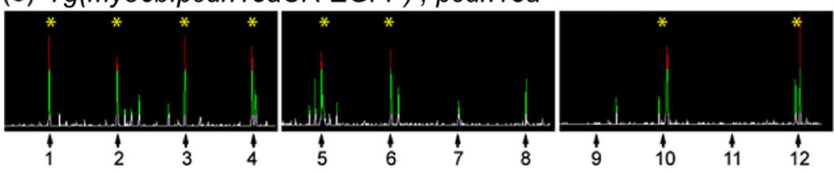

(f) $\operatorname{Tg}($ myo6b:pcdh15aCD1-EGFP) ; pcdh15ath263b

B

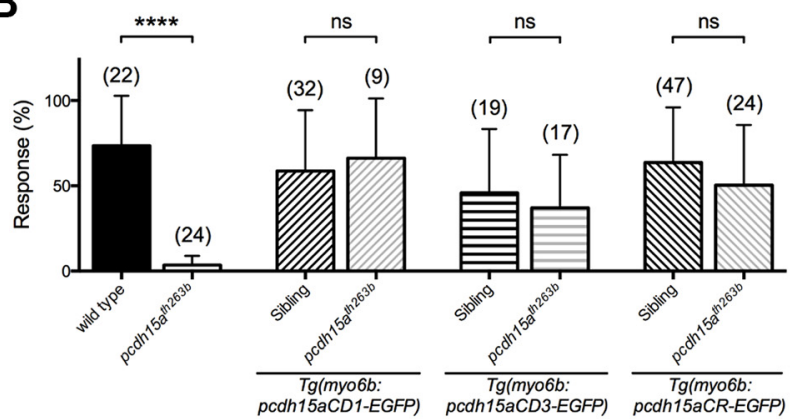

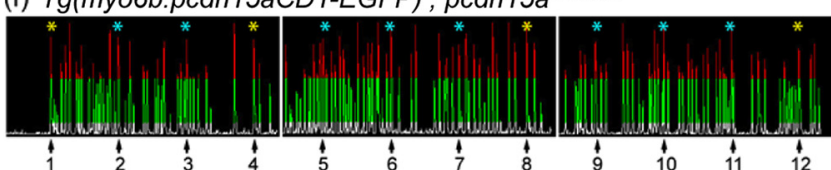

C

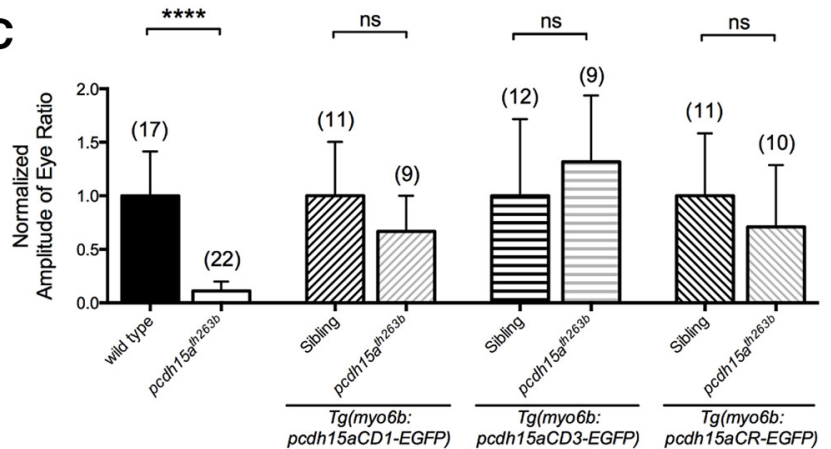

Figure 4. Rescue of behavioral defects in $p c d h 15 a^{\text {th } 263 \mathrm{~b}}$ mutants by either isoform of Pcdh $15 \mathrm{a}$. $A$, Representative time course of the AEBR to a pure tone with $1 \mathrm{kHz}$ at $157 \mathrm{~dB}$ at $5 \mathrm{dpf}$. The stimulus was repeated every $15 \mathrm{~s}$ for $3 \mathrm{~min}$. Numbered arrows indicate the stimuli and a yellow asterisk indicates a positive response. The blue asterisks indicate trials that were omitted from the data. The record shown in $A \boldsymbol{a}$ is an example of a wild-type control larva that responded to every stimulus, $\boldsymbol{A}$ f is a hyperactive larva, $\boldsymbol{A} \boldsymbol{b}$ is a homozygous mutant larvae, and $\boldsymbol{A c}$ through $\boldsymbol{A e}$ are rescue constructs as labeled. B, Startle responses of wild-type, $p c d h 15 a$ mutant, and rescued $p c d h 15$ a mutant larvae at $5 \mathrm{dpf}$ (number of fish is indicated above each bar). The mean and SD is indicated; $p$-values were determined by unpaired Mann-Whitney $U$ tests, ${ }^{* * *} p<0.0001$. C, Vestibular-induced eye movements in wild-type, $p c d h 15 a$ mutant, and rescued $p c d h 15 a$ mutant larvae at $5 \mathrm{dpf}$ (number of fish is indicated above each bar). The normalized peak amplitude of vestibular-induced eye movements at $0.25 \mathrm{~Hz}$ is shown. The mean and SD is indicated; $p$-values were determined by unpaired Mann-Whitney $U$ tests, ${ }^{* * * *} p<0.0001$.

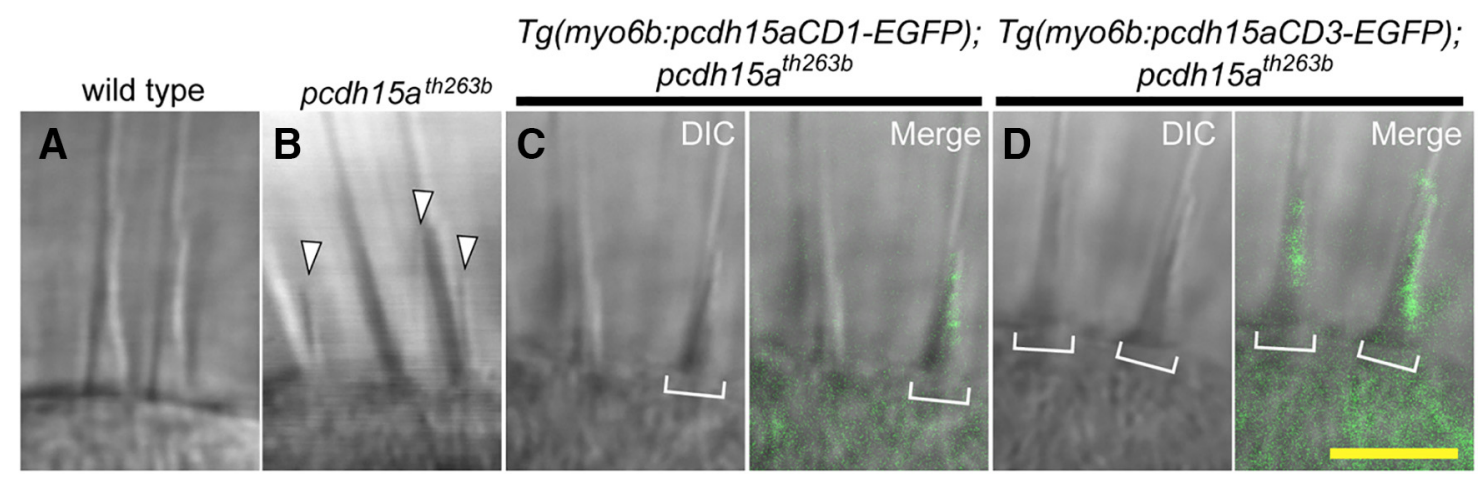

Figure 5. Rescue of the morphological defects of hair bundles in $p c d h 15 a^{\text {th263b }}$ mutants by either isoform of Pcdh15a. Images of inner ear hair cells in live fish were obtained at $6 \mathrm{dpf}$. $\boldsymbol{A}$, Wild-type hair bundles have a cone-shaped appearance. $\boldsymbol{B}, \operatorname{In}$ the $p c d h 15 a^{\text {th263b }}$ mutants, the hair bundles are splayed and split away from the kinocilia (arrowheads). $\boldsymbol{C}, \boldsymbol{D}$, Morphological defects of mutant hair bundles were rescued with Pcdh15a-CD1-EGFP (C) or Pcdh15a-CD3-EGFP (D). Brackets indicate hair bundles with detectable GFP signal. $n>8$ larvae for each genotype. Yellow scale bar, $5 \mu \mathrm{m}$.

Both isoforms of Pcdh15a rescue FM4-64 labeling in pcdh15a mutant hair cells

We next sought to assess mechanotransduction in pcdh15a mutant larvae expressing our transgenes. Vital dyes such as FM1-43 and FM4-64 can rapidly permeate hair cells through functional mechanotransduction channels (Meyers et al., 2003). Therefore,
FM dye labeling is commonly used as a proxy for mechanotransduction in mature hair cells (Meyers et al., 2003; Kindt et al., 2012). In both PCDH15-deficient Ames waltzer ${ }^{\text {av3J }}$ mice and $p c d h 15 a$ zebrafish mutants, hair cells do not accumulate FM1-43 dye in the soma (Seiler and Nicolson, 1999; Senften et al., 2006). 


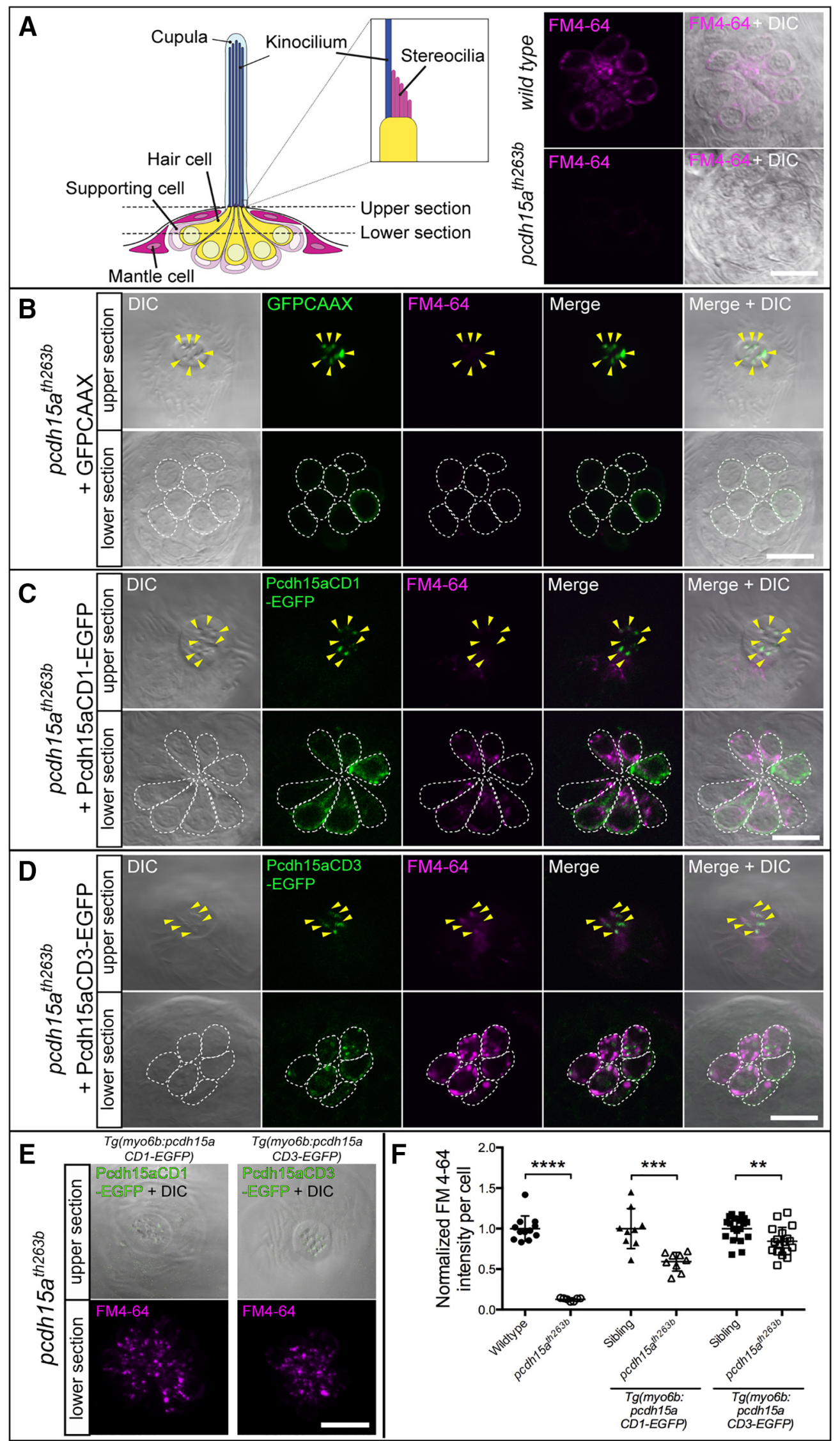

Figure 6. Rescue of defective FM4-64 labeling of $p c d h 15 a^{\text {th263b }}$ mutant hair cells by either isoform of Pcdh15a. $A$, Left, Diagram indicating focal planes of neuromast hair cells used for imaging of the GFP signal and FM4-64 label in wild-type and $p c d h 15 a^{\text {th263b }}$ larvae. Top and bottom sections in B-D show hair bundles and cell bodies, respectively. $\boldsymbol{A}$, Right, FM4-64 labeling in wild-type (top) and $p c d h 15 a^{\text {th263b }}$ mutant hair cells (bottom), 4 dpf. $\boldsymbol{B}-\boldsymbol{D}$, Representative images of the FM4-64 label in neuromasts of $4 \mathrm{dpf} p c$ dh15a ${ }^{\text {th263b }}$ larvae with (Figure legend continues.) 
To investigate functional differences between Pcdh15a-CD1 and Pcdh15a-CD3, we injected plasmid DNA encoding pcdh15aCD1-EGFP or pcdh15a-CD3-EGFP, driven by the hair-cellspecific $m y o 6 b$ promoter, into $p c d h 15 a^{\text {th263b }}$ mutant eggs and raised them to the larval stage. Injection of plasmid DNA led to transient mosaic expression with only a subset of hair cells expressing the EGFP-tagged proteins (Figs. 6, 7, 8). Using mosaic analysis, we could compare the level of rescue to nonrescued neighboring cells directly. For our experiments, we imaged lateral line hair cells, which are readily accessible to vital dyes in intact larvae, and we used FM4-64, which has nonoverlapping emission spectra with EGFP. It was reported that FM4-64 can label hair cells with a time course similar to FM1-43 (Meyers et al., 2003). As expected, we observed a significant reduction of FM4-64 labeling in $p c d h 15 a^{\text {th263b }}$ mutant hair cells compared with wildtype hair cells at $4 \mathrm{dpf}$ (Fig. 6A). The background fluorescence was not subtracted in our images and the remaining signal in the mutants likely reflects autofluorescence or the presence of negligible amounts of FM label within the outer leaflet of the apical plasma membrane. As a control experiment, we used EGFP fused with a CAAX prenylation site. Prenylation of the CAAX site targets EGFP to the plasma membrane, which leads to an enrichment of the signal in hair bundles (Fig. 6B, top). pcdh15a-mutant hair cells expressing EGFPCAAX did not label with FM4-64 dye (Fig. 6B, bottom). Upon expression of pcdh15a-CD1-EGFP or pcdh15a-CD3-EGFP, EGFP-positive hair cells were brightly labeled with FM4-64 (Fig. 6C,D). These results are consistent with those observed with the behavioral analyses.

Mosaic expression is useful for demonstrating effects among cells within the same neuroepithelium; however, transient expression of a gene can be variable due to several factors, including differences in the amount of plasmid injected and the number of transgenes that are integrated within each cell. To avoid issues associated with transient expression, we quantified FM labeling in stable transgenic lines. Although positional effects can lead to differences in expression levels between transgenic lines, the expression level of a particular transgene is more consistent. The $p c d h 15 a^{\text {th263b }}$ mutation is normally lethal when homozygous, with death occurring at $9 \mathrm{dpf}$. However, we were able to raise homozygous $p c d h 15 a^{\text {th } 263 \mathrm{~b}}$ mutants to adulthood upon stable expression of the pcdh15a-CD1-EGFP, pcdh15a-CD3-EGFP, or $p c d h 15 a-C R-E G F P$ transgenes. In contrast, we were unable to identify homozygous mutant adults carrying the other $p c d h 15 a$ transgenes with various deletions described in Figure $7 A$.

We compared transgenic and nontransgenic siblings generated by crossing transgenic homozygous or heterozygous $p c d h 15 a^{\text {th263b }}$ fish to nontransgenic th $263 b$ heterozygous fish. These crosses yielded mutant fish with or without the transgene. Consistent with the mosaic expression experiments, FM4-64 dye

\section{$\leftarrow$}

(Figure legend continued.) transient expression of GFPCAAX (B), pcdh15a-CD1-EGFP (C), or pcdh15a-CD3-EGFP (D). Arrowheads indicate EGFP-positive hair bundles. Somas of individual hair cells are outlined by white dotted lines. $\boldsymbol{E}$, Representative images of rescued mutant hair cells in $C D 1$ and $C D 3$ stable transgenic lines, $6 \mathrm{dpf}$. The bottom section is a maximum projection of seven sections (the same as those used in $\boldsymbol{F}$ for quantification). $\boldsymbol{F}$, Quantification of the intensity of FM4-64 labeling of EGFP-positive hair cells in stable transgenic lines. The values of the nontransgenic and transgenic homozygous mutants are normalized to the mean value of their corresponding wild-type siblings. For each transgenic construct, $n \geq 9$ neuromasts from a minimum of three larvae. Nontransgenic mutants $(n=7)$ and their wild-type siblings $(n=12)$ are from the $p c d h 15 a-C D 3-E G F P$ line. The mean and SD are indicated; $p$-values were determined by unpaired Student's $t$ tests (two-tailed, Welch-corrected), ${ }^{* *} p<0.01$; ${ }^{* * *} p<0.001$; ${ }^{* * * *} p<0.0001$. Scale bars in $\boldsymbol{A}-\boldsymbol{E}, 10 \mu \mathrm{m}$. was present in the soma of mutant hair cells stably expressing either Pcdh15a isoform (Fig. 6E). Overall, the level of FM labeling in the rescued mutants was lower compared with wild-type siblings for each transgene. To determine whether gene dosage had an effect on the levels of FM4-64 in hair cells, we compared the normalized means of FM4-64 intensity in mutant larvae expressing one versus two copies of the pcdh15a-CD3-GFP transgene and the means were almost identical (single copy mean $=$ $0.85, n=12$; double copy mean $=0.84, n=18$ ). This result suggests that the transgenic constructs are likely expressed at saturating levels. We also never observed any significant difference in FM4-64 labeling between nontransgenic and transgenic wildtype sibling groups (CD1: nontransgenic mean $=1.15, n=9$; transgenic mean $=1.10, n=6$; CD3: nontransgenic mean $=$ $0.51, n=19$; versus transgenic mean $0.53, n=12$ ), suggesting that overexpression of the transgenes does not adversely affect the mechanotransduction complex or bundle environment. Although not directly comparable to the CD3 transgene, the tagged version of CD1 appeared to be less effective in rescuing FM labeling of hair cells (Fig. $6 F$ ). Why this is the case and whether CD1 plays a less important role in the lateral line organ requires further investigation. Nevertheless, our experiments suggest that either isoform alone can rescue mechanotransduction in pcdh15a-null mutants, albeit not to the same level as in wild-type siblings.

\section{CD1- or CD3-specific regions are not required for the localization and function of Pcdh15a in hair cells}

Because both isoforms have the ability to rescue behavioral responses, hair bundle defects, and mechanotransduction, we hypothesized that the CD1- or CD3-specific regions are not strictly required for the function of Pcdh15a in hair cells. To test this hypothesis, we engineered a truncated form of Pcdh15a that retains the common region of both isoforms, but does not have the CD1- or CD3-specific regions (Pcdh15a-CR-EGFP; Fig. 7A). As with the EGFP-tagged CD1 and CD3 transgenes, we examined the ability of Pcdh15a-CR-EGFP to localize to the hair bundle and to rescue the behavioral and mechanotransduction defects in $p c d h 15 a^{\text {th } 263 \mathrm{~b}}$ mutants. In a stable transgenic line, Pcdh15a-CREGFP was sufficient for rescue of auditory and vestibular reflexes in mutant fish (Fig. $4 A e, B, C$ ). In $p c d h 15 a^{\text {th263b }}$ hair cells, imaging showed that transiently or stably expressed Pcdh15a-CR-EGFP localizes in a punctate pattern at the tips of hair bundles like wild-type Pcdh15a (Fig. 7C,D) and could rescue the splaying of mutant pcdh15a hair bundles (Fig. 7B). Likewise, Pcdh15a-CREGFP was present in the hair bundle of lateral line hair cells (Fig. $8 A$, top). Furthermore, FM4-64-labeling experiments showed that Pcdh15a-CR-EGFP significantly restored mechanotransduction in $p c d h 15 a^{\text {th263b }}$ mutant hair cells (Fig. $\left.8 A, E, F\right)$. Like the full-length Pcdh15a transgenes, the intensity of FM4-64 labeling was not fully rescued by Pcdh15a-CR-EGFP compared with wildtype siblings (Fig. 8F).

Both the CR and TMD of PCDH15 have been implicated in protein-protein interactions with members of the mechanotransduction complex in hair cells (Xiong et al., 2012; Maeda et al., 2014; Zhao et al., 2014). To further characterize the in vivo relevance of these protein-binding motifs in Pcdh15a, we examined the localization and functionality of truncated and chimeric forms of Pcdh15a. $\operatorname{Pcdh} 15 \mathrm{a}(\Delta$ cyto)-EGFP lacks the entire cytoplasmic domain including the CR (Fig. 7A). Similar to full-length Pcdh15a, $\operatorname{Pcdh} 15 \mathrm{a}(\Delta$ cyto)-EGFP localized at the tips of hair bundles and could rescue splaying at 4 and $6 \mathrm{dpf}$ (Figs. 7E, F, 8B). We also observed the EGFP signal within kinocilia in a subset of hair cells (Fig. 
A

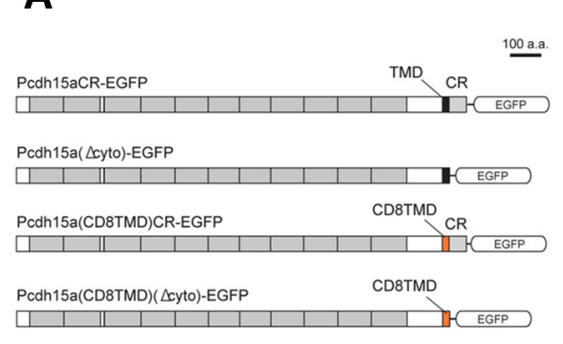

Transient Expression

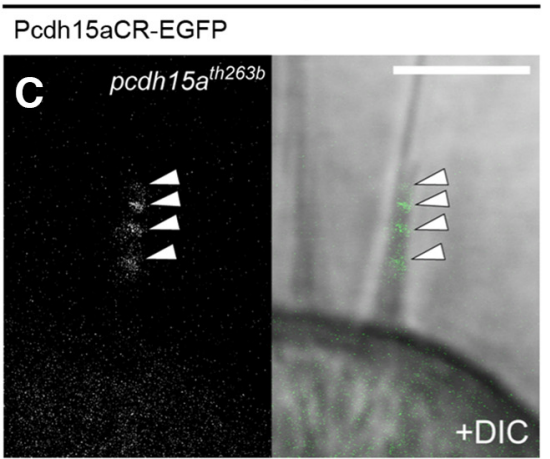

Pcdh15a( $\Delta$ cyto)-EGFP

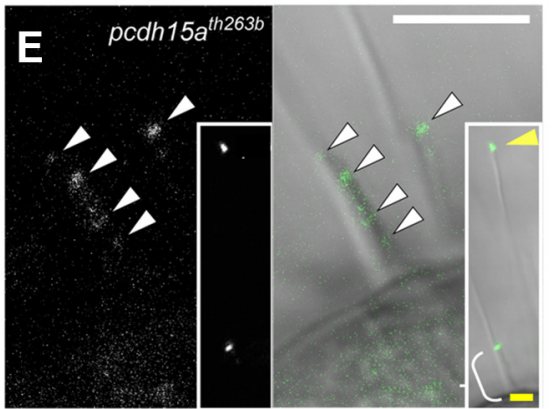

Pcdh15a(CD8TMD)CR-EGFP

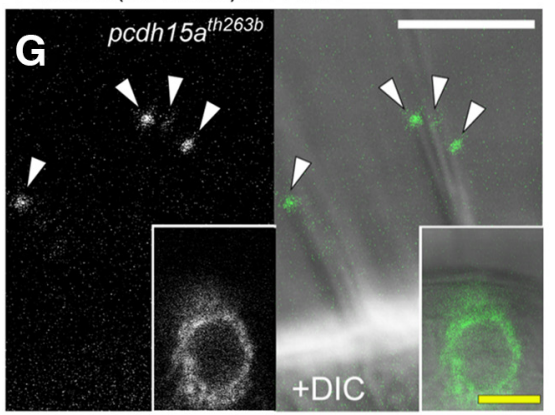

Pcdh15a(CD8TMD)(Acyto)-EGFP

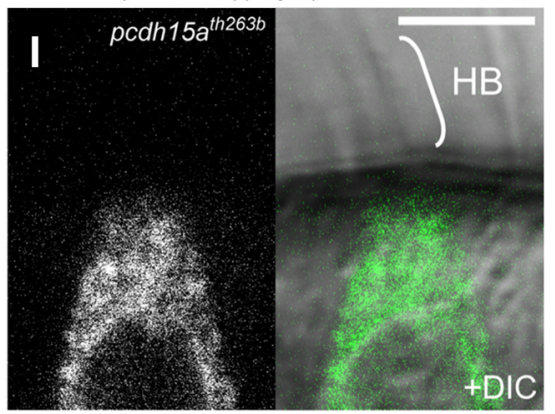

B

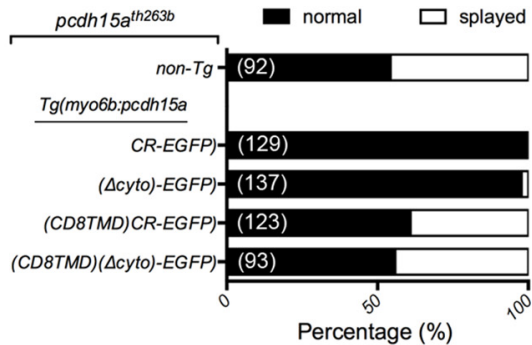

Stable Expression

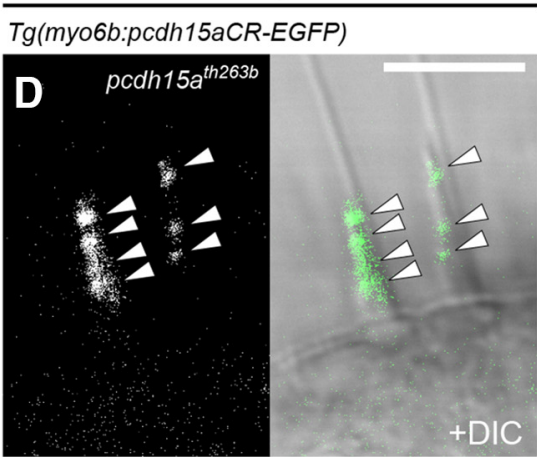

Tg(myo6b:pcdh15a(Acyto)-EGFP)

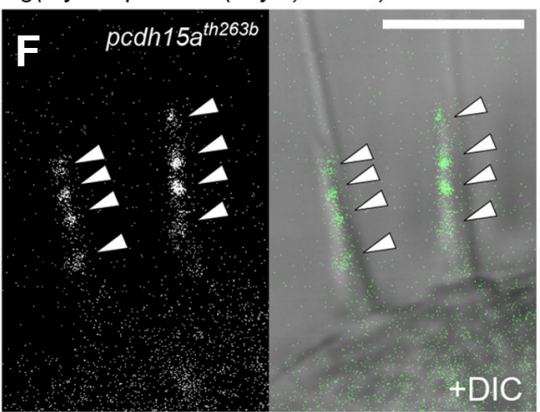

$T g$ (myo6b:pcdh15a(CD8TMD)CR-EGFP)

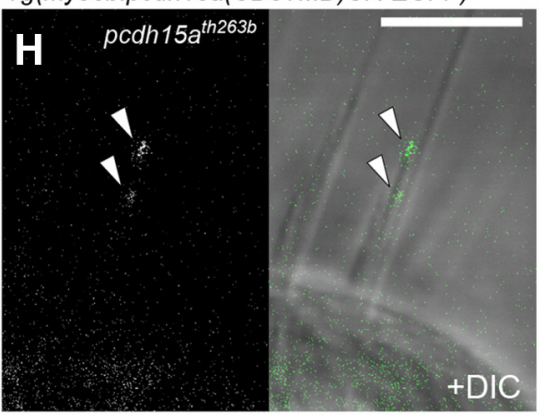

Tg(myo6b:pcdh15a(CD8TMD)( $\Delta c y t o)-E G F P)$

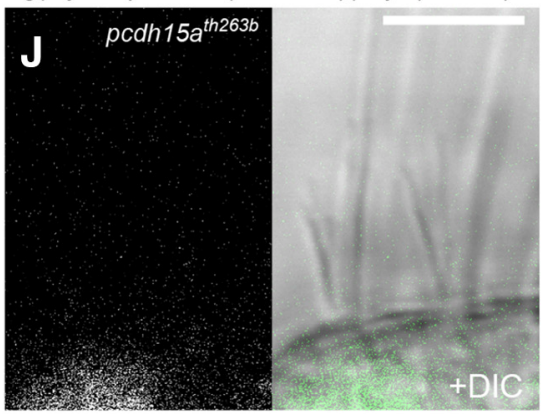

Figure 7. TMD and CR are required for localization of Pcdh15a to hair bundles and the TMD is critical for the rescue of splayed hair bundles in $p c d h 15 a^{\text {th263b }}$ mutants. $A$, Diagram of the protein constructs of Pcdh15a-CR-EGFP, Pcdh15a( $\Delta$ cyto)-EGFP, Pcdh15a(CD8TMD)CR-EGFP, and Pcdh15a(CD8TMD)( $\Delta$ cyto)-EGFP. $\boldsymbol{B}$, Percentage of normal (black) and splayed (white) hair

$7 E$, inset; $13 \%, n=38$ hair cells). This pattern suggests that the CR is partially required for retention at the site where the hair bundle connects to the kinocilium. Alternatively, this version of Pcdh15a may be incorrectly trafficked to the tip of the kinocilium. Experiments with larvae stably expressing $\operatorname{Pcdh} 15 \mathrm{a}(\Delta$ cyto $)$-EGFP showed that this truncated form was not able to restore FM4-64 labeling in $p c d h 15 a^{\text {th263b }}$ mutant hair cells above that of nontransgenic mutant siblings (Fig. $8 F$ ). These results indicate that the cytoplasmic common region is required for Pcdh15a function, but not for targeting Pcdh15a to the hair bundle.

To determine whether the TMD is required for Pcdh15a localization and function, we expressed a chimera, Pcdh15a (CD8TMD)-CR-EGFP, in which the TMD was replaced with the single-pass TMD from the type I protein CD8 (CD8TMD). Although the EGFP intensity was variable among fish, we observed localization of Pcdh15a(CD8TMD)-CR-EGFP in both the cell bodies and hair bundles of $p c d h 15 a^{\text {th263b }}$ mutants (Figs. $7 G, H, 8 C$ ). In $21 \%$ of transiently expressing hair cells, the hair bundles with Pcdh15a(CD8TMD)-CR-EGFP at the tips of stereocilia were still splayed (for example, see Fig. $7 G ; n=57$ ). In mutant larvae stably expressing Pcdh15a(CD8TMD)-CREGFP, splaying was slightly reduced (39\% vs $46 \%$ for nontransgenic mutants; Fig. $7 B$ ), but FM4-64 labeling was not restored (Fig. $8 F)$. These results suggest that the TMD is required for Pcdh15a function and is partially required for the trafficking of Pcdh15a into the hair bundles and for hair bundle integrity. Finally, we tested a CD8 chimeric protein that lacks both the entire cytoplasmic domain and the endogenous TMD (Pcdh15a(CD8TMD)( $\Delta$ cyto)-EGFP). The

\section{$\leftarrow$}

bundles. Top bar indicates the level of splaying in nontransgenic mutants. Bottom bars represent mutants with stable expression of each construct shown in $A$. The total number of hair bundles examined is indicated in parentheses. $n \geq 4$ larvae for each genotype. $(-\boldsymbol{J}$, Representative live images of transiently (left images, $4 \mathrm{dpf}$ ) and stably (right images, $6 \mathrm{dpf}$ ) expressed truncated and chimeric $P c d h 15$ a proteins in $p c d h 15^{\text {th263b }}$ mutants. C,D, Pcdh15a-CR-EGFP localized at the tips of hair bundles in a staircase-like manner (arrowheads). $\boldsymbol{E}, \boldsymbol{F}, \mathrm{P}$ cdh15a( $\Delta$ cyto)-EGFP localized at the tip of hair bundles, similar to Pcdh15a-CR-EGFP (white arrowheads). Inset in $\boldsymbol{E}, \operatorname{Pcdh} 15 \mathrm{a}(\Delta$ cyto)-EGFP was detected at the distal ends of kinocilia in 5 of 38 hair bundles (example indicated by yellow arrowhead; yellow scale bar in inset, $2.5 \mu \mathrm{m}$ ). Bracket indicates hair bundle. $\boldsymbol{G}, \boldsymbol{H}$, Pcdh15a(CD8TMD)-CR-EGFP localized at the tips of stereocilia (arrowheads). Note that the stereocilia are splayed in $\boldsymbol{G}$. Inset shows an example of GFP signal within the soma (scale bar, $5 \mu \mathrm{m}) . I, J$, Pcdh15a(CD8TMD)( $\Delta$ cyto)EGFP was absent in hair bundles. Bracket in $I$ indicates a hair bundle (HB). White scale bars in $C-J, 5 \mu \mathrm{m}$. 


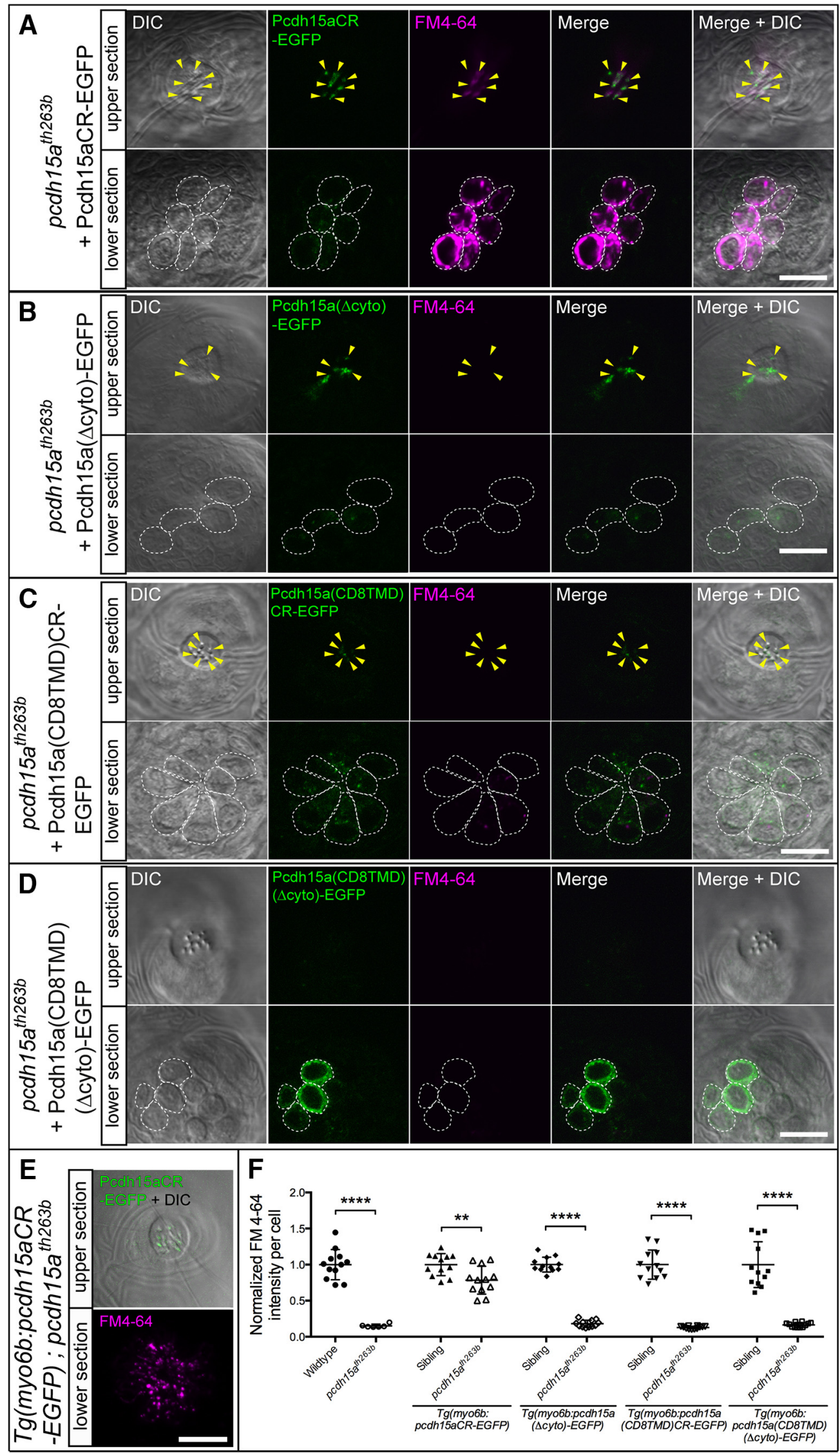

Figure 8. The CR and TMD are essential for the function of Pcdh15a in hair cells. $\boldsymbol{A}-\boldsymbol{D}$, Rescue of defective FM4-64 labeling of neuromast hair cells of $p c d h 15^{\text {th263b }}$ mutants with transient expression of truncated or chimeric Pcdh15a-EGFP (see diagram in Fig. 7A) at 4 dpf. FM4-64 labeling is shown in the presence of Pcdh15a-CR-EGFP $(\boldsymbol{A})$, Pcdh15a( $\Delta$ cyto)-EGFP $(\boldsymbol{B})$, Pcdh15a(CD8TMD)-CR-EGFP $(\boldsymbol{C})$, and Pcdh15a(CD8TMD)( $\Delta$ cyto)-EGFP $(\boldsymbol{D})$. Arrowheads indicate the EGFP-positive hair bundles. Top and bottom sections are (Figure legend continues.) 
Pcdh15a(CD8TMD)( $\Delta$ cyto)-EGFP signal was almost exclusively in the soma of $p c d h 15 a^{\text {th } 263 \mathrm{~b}}$ mutants (Figs. $7 I, J, 8 D$ ). Splaying of hair bundles still occurred at levels comparable to $p c d h 15 a^{\text {th } 263 \mathrm{~b}}$ mutants (44\%; Fig. 7B). These data suggest that the proper trafficking of Pcdh15a to the tips of stereocilia requires both the CR and the TMD. Consistent with the inability of Pcdh15a(CD8TMD)( $\Delta$ cyto)-EGFP to correctly localize in the hair bundle, stable expression of this construct was unable to restore FM4-64 label above that observed in the nontransgenic mutants (Fig. 8F). Collectively, this series of experiments demonstrate that, together with the extracellular domain, the TMD and CR of Pcdh15a are necessary and sufficient for the localization of Pcdh15a in the hair bundle and that both domains are critical for the function of Pcdh15a in mediating mechanotransduction.

\section{Discussion}

PCDH15 is a tip link protein that is essential for mechanotransduction in hair cells (Ahmed et al., 2006; Kazmierczak et al., 2007; Alagramam et al., 2011). Along with CDH23, the extracellular cadherin repeats of PCDH15 form part of the filamentous tip link; together as a trans-adhesive complex, these unusually long cadherins span the distance between neighboring stereocilia $(\mathrm{Ka}-$ zmierczak et al., 2007). In contrast to the extracellular domain of PCDH15, the function of the divergent intracellular domains of PCDH15 is not as clear. In this study, we exogenously expressed EGFP-tagged versions of zebrafish Pcdh15a-CD1 and Pcdh15aCD3 in pcdh15a-null mutants to assess their localization and functionality. Although imaging of tip links in a cochlear explant has been reported using hopping probe ion conductance microscopy (Novak et al., 2009), imaging of a tip link protein in an intact animal is unprecedented; to date, a fluorescently tagged version of mammalian PCDH15 expressed in hair cells has not been reported. Our pcdh15a-EGFP transgenic lines provide an important tool for visualizing a central component of the transduction complex in zebrafish hair cells during development and in disease models associated with loss of hair cell function.

We found that, despite the divergence of the CD1 and CD3 isoforms of Pcdh15a, both isoforms localize to the tips of stereocilia and are capable of restoring function to $p c d h 15 a$ mutant hair cells; transgenic expression of either Pcdh15a-CD1-EGFP or Pcdh15a-CD3-EGFP alone can rescue the morphological and functional defects in a $p c d h 15 a$-null mutant. Furthermore, rescue experiments with C-terminally truncated and chimeric forms of Pcdh15a demonstrate that the TMD and intracellular CR of Pcdh15a both contribute to Pcdh15a localization and function. Our experiments here provide the first in vivo evidence that these domains are key to the function of Pcdh15a in hair cells.

The cytoplasmic tail of PCDH15 has been shown to interact in vitro or in heterologous experiments with a number of proteins implicated in mechanotransduction, including LHFPL5, MYO7A, and a PDZ-containing protein, USH1C (also known as

\footnotetext{
$\leftarrow$

(Figure legend continued.) included as in Figure 6.E, Representative image of rescued mutant hair cells in a Pcdh15a-CR-EGFP stable transgenic line. Bottom section is a maximum projection of seven sections (the same as those used in $\boldsymbol{F}$ for quantification). $\boldsymbol{F}$, Quantification of the intensity of FM4-64 labeling of EGFP-positive hair cells in stable transgenic lines. The values of the nontransgenic and transgenic homozygous mutants are normalized to the mean value of their corresponding wild-type siblings. For each transgenic construct, $n \geq 9$ neuromasts from a minimum of three larvae. Nontransgenic mutants $(n=6)$ and their wild-type siblings $(n=12)$ are from the $p c d h 15 a(\Delta c y t)$-EGFP line. The mean and SD are indicated; $p$-values were determined by unpaired Student's t tests (two-tailed, Welch-corrected), ${ }^{* *} p<0.01$; ${ }^{* * *} p<0.001$; ${ }^{* * * *} p<0.0001$. Scale bars in $\boldsymbol{A}-\boldsymbol{E}, 10 \mu \mathrm{m}$.
}

Harmonin) (El-Amraoui and Petit, 2005; Yan and Liu, 2010; Zheng et al., 2012; Xiong et al., 2012). In mice, mutations or deletions of Lhfpl5, Myo7a, and Ush1c cause the reduction or mislocalization of PCDH15 in stereocilia (Senften et al., 2006; Yan et al., 2011; Xiong et al., 2012). In agreement with previous findings, we observed that Pcdh15-CD3-EGFP is absent at the tips of mature hair bundles in $\operatorname{lhfpl} 5 a$, myo $7 a a$, and $c d h 23$ mutant fish. Like $c d h 23$ mutants, myo7aa mutants displayed robust EGFP signal in immature hair bundles, but not in more mature hair bundles. In contrast, EGFP-tagged Pcdh15a remained in the hair cell body of $\operatorname{lhfpl} 5 \mathrm{a}$ mutants at all developmental stages, implying that Lhfpl5a is required for transport to the hair bundle. A similar role was reported for the mammalian ortholog of $\operatorname{lhfpl} 5 \mathrm{a}$ (Xiong et al., 2012), suggesting that the trafficking function of LHFPL5 is a conserved feature in vertebrates.

The motifs of PCDH15 that are essential for targeting or stable localization of PCDH15 to the site of mechanotransduction have not been previously described. Our findings with Pcdh15a-CR-EGFP show that the CD1- and CD3-specific regions are not required for the correct localization of Pcdh15a. Moreover, the CR is also not required for Pcdh15a to localize at the tips of stereocilia. That the CR is not strictly required for localization suggests that formation of a trans-adhesive complex with Cdh23 or interactions with the intact TMD region are sufficient for trafficking of Pcdh15a to the tips of stereocilia. The TMD chimera Pcdh15a(CD8TMD)-CR-EGFP was also present in hair bundles, albeit in a more variable pattern, with more Pcdh15a(CD8TMD)-CR-EGFP protein retained in the cell body. However, if the cytoplasmic domain was deleted from the TMD chimera, then the protein was completely absent in hair bundles in the null mutant background, suggesting that, along with the trans-adhesive complex with $\mathrm{Cdh} 23$, the combined region of the TMD and CR is critical for the trafficking and proper localization of Pcdh15a. It is likely that these domains contribute to the trafficking of Pcdh15a via direct interactions with Lhfpl5a and perhaps via interactions with Myo7aa, as was demonstrated for the mouse orthologous proteins LHFPL5 and MYO7A in cell line experiments (Senften et al., 2006; Xiong et al., 2012).

In regard to extracellular motifs, the N-terminal cadherin repeats of PCDH15 mediate the heterophilic interaction with the $\mathrm{N}$ terminus of CDH23 (Elledge et al., 2010; Sotomayor et al., 2012; Geng et al., 2013). In hair cell explants undergoing regeneration of tip links, PCDH15 homomeric interactions occur transiently, followed by a subsequent switch to the PCDH15-CDH23 heteromeric complex (Indzhykulian et al., 2013). In Cdh23 mutant mice, immunolabeling of the CD1 isoform of PCDH15 was reported to be unaffected in cochlear hair cells at postnatal day 5 (Senften et al., 2006). Our results indicate that the zebrafish CD3 isoform is initially present in immature $c d h 23$ mutant hair bundles, possibly as homomeric complexes. However, within more mature hair bundles, Pcdh15a-CD3-EGFP was absent in $c d h 23$ mutants. The absence of tagged-Pcdh15a-CD3 in mature cells suggests that this trans-adhesive complex is required for retaining Pcdh15a-CD3 in the hair bundle.

Of the various constructs, the loss of the entire cytoplasmic domain of Pcdh15a was the only mutated form that we observed in the tips of kinocilia. Previous studies have shown an asymmetry of the kinocilial link, with PCDH15 located in the kinocilium (Goodyear et al., 2010; Lelli et al., 2010). The CR may be partially required for retention of $\mathrm{Pcdh} 15 \mathrm{a}$ at this site within kinocilia; nevertheless, kinocilial links appeared to be intact in $p c d h 15 a$ mutants expressing the Pcdh $15 \mathrm{a}(\Delta$ cyto)-EGFP construct. In addition, the $\operatorname{Pcdh} 15 \mathrm{a}(\Delta$ cyto)-EGFP construct was the only construct that fully rescued the splayed bundle phenotype, but 
not function. This result suggests that the CR is key to functional coupling of the tip link to the transduction machinery.

Interestingly, we found that Pcdh15a could localize to the tips of splayed or disorganized hair bundles. Although variable with regard to localization, Pcdh15a(CD8TMD)-CR-EGFP was detected at the tips of stereocilia in $p c d h 15 a$ mutant hair cells even if the stereocilia were split away from the bundle. Localization of Pcdh15a-CD3-EGFP also persisted in disorganized hair bundles in myo6b mutant fish. Collectively, our results and previous studies suggest that multiple components are required for localization of Pcdh15a to the tips of stereocilia in hair cells, including the facilitation of transport to the hair bundle by Lhfpl5a and subsequent formation of a trans-complex with Cdh23.

Our study also underscores the importance of the CR and TMD for PCDH15 function. Previous yeast two-hybrid and heterologous expression experiments have shown that the interaction of PCDH15 with other components of the transduction complex such as TMC1, TMC2, and LHFPL5 is mediated through the CR and TMD (Maeda et al., 2014; Xiong et al., 2012). Indeed, in vivo experiments in zebrafish revealed that disruption of the CR-mediated Pcdh15a/Tmc interaction via overexpression of the $\mathrm{N}$ terminus of Tmc2a leads to mislocalization of Pcdh15a and decreased hair cell activity (Maeda et al., 2014). However, direct evidence that the CR and TMD are required for the function of PCDH15 has been lacking. We found that, in the absence of the CR, the basal activity of mechanotransduction channels is compromised, as evidenced by the inability of FM4-64 to enter hair cells. Aside from the requirement of the CR, the present study also suggests that the particular amino acid sequence of the Pcdh15a TMD is important for function. The multiple interactions mediated by the CR and TMD with members of the transduction complex suggest that PCDH15 serves as a hub or central component of the complex.

In mice, PCDH15-CD1, PCDH15-CD2, and PCDH15-CD3 are not uniquely required for tip link formation during development (Webb et al., 2011). In contrast, PCDH15-CD2 is an essential component of the tip link in mature cochlear hair cells (Pepermans et al., 2014). Although zebrafish do not have a Pcdh15a-CD2 isoform (Maeda et al., 2014), mammalian PCDH15-CD2 has taken on an indispensable, specialized role in mechanotransduction in mature cochlear hair cells. The C-terminal tail of the CD2 isoform was shown recently to interact with TMIE, a membrane protein required for auditory/vestibular function (Zhao et al., 2014). However, in mammalian vestibular hair cells, this interaction is not essential. Like mammalian vestibular hair cells, zebrafish hair cells do not require a CD2-Tmie interaction to form a functional mechanotransduction complex and our rescue experiments show that Pcdh15a-CR is sufficient for the formation and function of the mechanotransduction complex. However, our study does not rule out subtle effects caused by the deletion of the specific regions of CD1 and CD3. Future experiments examining the effects on transduction currents may reveal distinct functional differences between the isoform-specific regions. Although the Pcdh15a-CD1, Pcdh15a-CD3, and Pcdh15a-CR proteins were all able to fully restore vestibular and auditory behaviors, none of the transgenes tested here were able to restore FM4-64 labeling to wild-type levels. Nevertheless, our study is an important step toward understanding the role of the TMD and CR of Pcdh15a in vestibuloauditory and lateral line hair cells and reveals the molecular features of Pcdh15a that are likely to be conserved in vertebrates.

\section{References}

Ahmed ZM, Goodyear R, Riazuddin S, Lagziel A, Legan PK, Behra M, Burgess SM, Lilley KS, Wilcox ER, Riazuddin S, Griffith AJ, Frolenkov GI, Bely- antseva IA, Richardson GP, Friedman TB (2006) The tip-link antigen, a protein associated with the transduction complex of sensory hair cells, is protocadherin-15. J Neurosci 26:7022-7034. CrossRef Medline

Alagramam KN, Goodyear RJ, Geng R, Furness DN, van Aken AF, Marcotti W, Kros CJ, Richardson GP (2011) Mutations in protocadherin 15 and cadherin 23 affect tip links and mechanotransduction in mammalian sensory hair cells. PLoS One 6:e19183. CrossRef Medline

Assad JA, Shepherd GM, Corey DP (1991) Tip-link integrity and mechanical transduction in vertebrate hair cells. Neuron 7:985-994. CrossRef Medline

Beurg M, Fettiplace R, Nam JH, Ricci AJ (2009) Localization of inner hair cells mechnaotransducer channels using high-speed calcium imaging. Nat Neurosci 12:553-558. CrossRef Medline

Chen X, Zaro JL, Shen WC (2013) Fusion protein linkers: property, design and functionality. Adv Drug Deliv Rev 65:1357-1369. CrossRef Medline

Einhorn Z, Trapani JG, Liu Q, Nicolson T (2012) Rabconnectin3alpha promotes stable activity of the $\mathrm{H}+$ pump on synaptic vesicles in hair cells. J Neurosci 32:11144-11156. CrossRef Medline

El-Amraoui A, Petit C (2005) Usher I syndrome: unravelling the mechanisms that underlie the cohesion of the growing hair bundle in inner ear sensory cells. J Cell Sci 118:4593-4603. CrossRef Medline

Elledge HM, Kazmierczak P, Clark P, Joseph JS, Kolatkar A, Kuhn P, Müller U (2010) Structure of the $\mathrm{N}$ terminus of cadherin 23 reveals a new adhesion mechanism for a subset of cadherin superfamily members. Proc Natl Acad Sci U S A 107:10708-10712. CrossRef Medline

Erickson T, French CR, Waskiewicz AJ (2010) Meis1 specifies positional information in the retina and tectum to organize the zebrafish visual system. Neural Dev 5:22. CrossRef Medline

Ernest S, Rauch GJ, Haffter P, Geisler R, Petit C, Nicolson T (2000) Mariner is defective in myosin VIIA: a zebrafish model for human hereditary deafness. Hum Mol Genet 9:2189-2196. CrossRef Medline

Geng R, Sotomayor M, Kinder KJ, Gopal SR, Gerka-Stuyt J, Chen DH, Hardisty-Hughes RE, Ball G, Parker A, Gaudet R, Furness D, Brown SD, Corey DP, Alagramam KN (2013) Noddy, a mouse harboring a missense mutation in protocadherin-15, reveals the impact of disrupting a critical interaction site between tip-link cadherins in inner ear hair cells. J Neurosci 33:4395-4404. CrossRef Medline

Goodyear RJ, Richardson GP (2003) A novel antigen sensitive to calcium chelation that is associated with the tip links and kinocilial links of sensory hair bundles. J Neurosci 23:4878-4887. Medline

Goodyear RJ, Forge A, Legan PK, Richardson GP (2010) Asymmetric distribution of cadherin 23 and protocadherin 15 in the kinocilial links of avian sensory hair cells. J Comp Neurol 518:4288-4297. CrossRef Medline

Granato M, van Eeden FJ, Schach U, Trowe T, Brand M, Furutani-Seiki M, Haffter P, Hammerschmidt M, Heisenberg CP, Jiang YJ, Kane DA, Kelsh RN, Mullins MC, Odenthal J, Nüsslein-Volhard C (1996) Genes controlling and mediating locomotion behavior of the zebrafish embryo and larva. Development 123:399-413. Medline

Hudspeth AJ (1989) How the ear's works work. Nature 341:397-404. CrossRef Medline

Indzhykulian AA, Stepanyan R, Nelina A, Spinelli KJ, Ahmed ZM, Belyantseva IA, Friedman TB, Barr-Gillespie PG, Frolenkov GI (2013) Molecular remodeling of tip links underlies mechanosensory regeneration in auditory hair cells. PLoS Biol 11:e1001583. CrossRef Medline

Kazmierczak P, Sakaguchi H, Tokita J, Wilson-Kubalek EM, Milligan RA, Müller U, Kachar B (2007) Cadherin 23 and protocadherin 15 interact to form tip-link filaments in sensory hair cells. Nature 449:87-91. CrossRef Medline

Kindt KS, Finch G, Nicolson T (2012) Kinocilia mediate mechanosensitivity in developing zebrafish hair cells. Dev Cell 23:329-341. CrossRef Medline

Kwan KM, Fujimoto E, Grabher C, Mangum BD, Hardy ME, Campbell DS, Parant JM, Yost HJ, Kanki JP, Chien CB (2007) The Tol2kit: a multisite gateway-based construction kit for Tol2 transposon transgenesis constructs. Dev Dyn 236:3088-3099. CrossRef Medline

Lelli A, Kazmierczak P, Kawashima Y, Müller U, Holt JR (2010) Development and regeneration of sensory transduction in auditory hair cells requires functional interaction between cadherin- 23 and protocadherin- 15 . J Neurosci 30:11259-11269. CrossRef Medline

Longo-Guess CM, Gagnon LH, Cook SA, Wu J, Zheng QY, Johnson KR (2005) A missense mutation in the previously undescribed gene Tmhs underlies deafness in hurry-scurry (hscy) mice. Proc Natl Acad Sci U S A 102:7894-7899. CrossRef Medline 
Maeda R, Kindt KS, Mo W, Morgan CP, Erickson T, Zhao H, ClemensGrisham R, Barr-Gillespie PG, Nicolson T (2014) Tip-link protein protocadherin 15 interacts with transmembrane channel-like proteins TMC1 and TMC2. Proc Natl Acad Sci U S A 111:12907-12912. CrossRef Medline

Meyers JR, MacDonald RB, Duggan A, Lenzi D, Standaert DG, Corwin JT, Corey DP (2003) Lighting up the senses: FM1-43 loading of sensory cells through nonselective ion channels. J Neurosci 23:4054-4065. Medline

Mo W, Chen F, Nechiporuk A, Nicolson T (2010) Quantification of vestibular-induced eye movements in zebrafish larvae. BMC Neurosci 11:110. CrossRef Medline

Nicolson T, Rüsch A, Friedrich RW, Granato M, Ruppersberg JP, NüssleinVolhard C (1998) Genetic analysis of vertebrate sensory hair cell mechanosensation: the zebrafish circler mutants. Neuron 20:271-283. CrossRef Medline

Novak P, Li C, Shevchuk AI, Stepanyan R, Caldwell M, Hughes S, Smart TG, Gorelik J, Ostanin VP, Lab MJ, Moss GW, Frolenkov GI, Klenerman D, Korchev YE (2009) Nanoscale live-cell imaging using hopping probe ion conductance microscopy. Nat Methods 6:279-281. CrossRef Medline

Obholzer N, Wolfson S, Trapani J, Mo W, Nechiporuk A, Busch-Nentwich E, Seiler C, Sidi S, Soellner C, Duncan R, Boehland A, Nicolson T (2008) Vesicular Glutamate Transporter 3 is required for synaptic transmission in zebrafish hair cells. J Neurosci 28:2110-2118. CrossRef Medline

Pepermans E, Michel V, Goodyear R, Bonnet C, Abdi S, Dupont T, Gherbi S, Holder M, Makrelouf M, Hardelin JP, Marlin S, Zenati A, Richardson G, Avan P, Bahloul A, Petit C (2014) The CD2 isoform of protocadherin-15 is an essential component of the tip-link complex in mature auditory hair cells. EMBO Mol Med 6:984-992. CrossRef Medline

Seiler C, Nicolson T (1999) Defective calmodulin-dependent rapid apical endocytosis in zebrafish sensory hair cell mutants. J Neurobiol 41:424434. Medline

Seiler C, Ben-David O, Sidi S, Hendrich O, Rusch A, Burnside B, Avraham $\mathrm{KB}$, Nicolson T (2004) Myosin VI is required for structural integrity of the apical surface of sensory hair cells in zebrafish. Dev Biol 272:328-338. CrossRef Medline

Seiler C, Finger-Baier KC, Rinner O, Makhankov YV, Schwarz H, Neuhauss SC, Nicolson T (2005) Duplicated genes with split functions: independent roles of protocadherin 15 orthologues in zebrafish hearing and vision. Development 132:615-623. CrossRef Medline

Senften M, Schwander M, Kazmierczak P, Lillo C, Shin JB, Hasson T, Géléoc GS, Gillespie PG, Williams D, Holt JR, Müller U (2006) Phys- ical and functional interaction between protocadherin 15 and myosin VIIa in mechanosensory hair cells. J Neurosci 26:2060-2071. CrossRef Medline

Shabbir MI, Ahmed ZM, Khan SY, Riazuddin S, Waryah AM, Khan SN, Camps RD, Ghosh M, Kabra M, Belyantseva IA, Friedman TB, Riazuddin S (2006) Mutations of human TMHS cause recessively inherited nonsyndromic hearing loss. J Med Genet 43:634-640. CrossRef Medline

Sheets L, Trapani J, Mo W, Obholzer N, Nicolson T (2011) Ribeye is required for presynaptic Cav1.3a channel localization and afferent innervation of sensory hair cells. Development 138:1309-1319. CrossRef Medline

Siemens J, Lillo C, Dumont RA, Reynolds A, Williams DS, Gillespie PG, Müller U (2004) Cadherin 23 is a component of the tip link in hair-cell stereocilia. Nature 428:950-955. CrossRef Medline

Söllner C, Rauch GJ, Siemens J, Geisler R, Schuster SC, Müller U, Nicolson T, Tubingen Screen C (2004) Mutations in cadherin 23 affect tip links in zebrafish sensory hair cells. Nature 428:955-959. CrossRef Medline

Sotomayor M, Weihofen WA, Gaudet R, Corey DP (2012) Structure of a force-conveying cadherin bond essential for inner-ear mechanotransduction. Nature 492:128-132. CrossRef Medline

Thisse C, Thisse B (2008) High-resolution in situ hybridization to wholemount zebrafish embryos. Nat Protoc 3:59-69. CrossRef Medline

Webb SW, Grillet N, Andrade LR, Xiong W, Swarthout L, Della Santina CC, Kachar B, Müller U (2011) Regulation of PCDH15 function in mechanosensory hair cells by alternative splicing of the cytoplasmic domain. Development 138:1607-1617. CrossRef Medline

Xiong W, Grillet N, Elledge HM, Wagner TF, Zhao B, Johnson KR, Kazmierczak P, Müller U (2012) TMHS is an integral component of the mechanotransduction machinery of cochlear hair cells. Cell 151:1283-1295. CrossRef Medline

Yan D, Kamiya K, Ouyang XM, Liu XZ (2011) Analysis of subcellular localization of Myo7a, Pcdh15 and Sans in Ush1c knockout mice. Int J Exp Pathol 92:66-71. CrossRef Medline

Yan D, Liu X (2010) Genetics and pathological mechanisms of Usher syndrome. J Hum Gen 55:327-335. CrossRef Medline

Zhao B, Wu Z, Grillet N, Yan L, Xiong W, Harkins-Perry S, Müller U (2014) TMIE is an essential component of the mechanotransduction machinery of cochlear hair cells. Neuron 84:954-967. CrossRef Medline

Zheng QY, Scarborough J, Zheng Y, Heping Y, Choi D, Gillespie P (2012) Digenic inheritance of deafness caused by $8 \mathrm{~J}$ allele of myosin-VIIA and mutations in other Usher 1 genes. Hum Mol Gen 21:2588-2598. CrossRef 